Pure and Applied Mathematics Quarterly

Volume 6, Number 2

(Special Issue: In honor of

Michael Atiyah and Isadore Singer)

457 - 500, 2010

\title{
Schwarzschild Spacetime without Coordinates
}

\author{
Orlando Alvarez
}

\begin{abstract}
We discuss how to construct the full Schwarzschild (KruskalSzekeres) spacetime in one swoop by using the bundle of orthonormal Lorentz frames and the Einstein equation without the use of coordinates. We never have to write down the Kruskal-Szekeres or an equivalent form of the metric. Keywords: general relativity, gravitation, Schwarzschild solution, frame bundles, global geometry.
\end{abstract}

\section{Contents}

$\begin{array}{ll}\text { Prologue } & 458\end{array}$

1. Introduction 459

2. Frame Bundles 461

3. Fibration 463

3.1. Local Description 465

3.2. Totally Geodesic Fibration 466

3.3. Gauss and Codazzi Equations 466

4. Riemannian Submersion 467

4.1. The Ricci Tensor and Ricci Scalar 472

5. The Group Action 472

Received December 31, 2007. 
5.1. Transitive Case and Invariant Tensors 472

5.2. General Case 473

5.3. The Basic Example 475

6. Spherically Symmetric $(3+1)$ Geometry 477

7. Vacuum Einstein Equations 482

7.1. Properties of the radius function 484

7.2. Geodesics 486

7.3. Schwarzschild Geometry without Coordinates 488

7.4. The Kruskal Spacetime 493

7.5. Schwarzschild Coordinates 494

7.6. Redshift without Coordinates 494

$\begin{array}{ll}\text { Acknowledgments } & 495\end{array}$

Appendix A. Cartan's Lemma 495

Appendix B. Lightcone Conventions 496

Appendix C. Cartan's Approach to Geodesics 496

C.1. Holonomy and Symmetric Spaces 498

$\begin{array}{ll}\text { References } & 499\end{array}$

\section{Prologue}

It is a great pleasure and a great honor to be a contributor to this volume celebrating Michael Atiyah and Isadore Singer. The work of Atiyah and Singer, especially their results on Index Theory, has greatly influenced my own.

Is Singer has been a close friend and colleague since 1982. It was he who explained to me the importance of principal bundles and their uses in global geometry. As a physicist I was "locally trained" in the use of local coordinates and local frames. It was Is who patiently taught me to think globally. This article 
uses the global geometry of the bundle of lorentzian frames, a principal bundle, to construct the maximal analytic extension of the Schwarzschild spacetime.

I thank Is for countless hours of discussion and most of all for his friendship.

\section{INTRODUCTION}

The Schwarzschild solution is probably the most studied nontrivial solution to the Einstein equations ${ }^{1}$. The exterior solution represents spherically symmetric stars and the interior has a black hole. What drives much of the intellectual curiosity of students of general relativity is the presence of the black hole and its consequences.

I have taught the general relativity course several times over a 20 year period and I have not been completely satisfied with the discussions of black holes in the introductory course. The presentation usually involves deriving the Schwarzschild metric in standard Schwarzschild coordinates then transforming to EddingtonFinkelstein coordinates to study what happens as one crosses the horizon and finally a discussion of the maximal extension in Kruskal-Szekeres coordinates, see for example $[2,3,4]$. Of course, you could take as a starting point the KruskalSzekeres solution. This is neither physically or mathematically satisfying because the radius $r$ of the symmetry 2-spheres is implicitly given in terms of the KruskalSzekeres $(T, R)$-coordinates by

$$
T^{2}-R^{2}=\left(1-\frac{r}{2 G M}\right) e^{r / 2 G M} .
$$

Another approach is to introduce a lot more mathematical machinery [5, 6] and discuss global causal structures and singularity structures of lorentzian manifolds but this is overkill if you just want to talk about the Schwarzschild solution.

I wanted to find a middle ground where you could see the whole extended Schwarzschild solution at once with the geometry and the physics transparent. In fact I wanted to find a coordinate independent way of describing the Schwarzschild solution. It began by trying to understand what Birkhoff's Theorem tells you about the bundle of Lorentz orthonormal frames. In the process I found such a coordinate independent geometric approach but unfortunately it is not elementary at the level of an introductory general relativity course. It requires much

\footnotetext{
${ }^{1}$ For a comprehensive study of known solutions to the Einstein equations see [1].
} 
more mathematics, especially an understanding of group actions on manifolds, of principal fiber bundles [7] and of riemannian submersions [8]. It is more than overkill, nevertheless, I believe the approach to be a new novel and insightful way of studying the Schwarzschild solution.

This article is not meant to be an exhaustive discussion of the Schwarzschild spacetime. I will pick and choose several topics that are of interest because of the mathematical methods I use. One glaring omission is the discussion of the actual singularity at $r=0$. The reason is that $\mathrm{I}$ have no new insight to offer.

In brief, the goal is to derive the extended Kruskal-Szekeres spacetime without ever writing coordinates. Instead of studying the geometry in the Schwarzschild spacetime $N$ directly, we work "upstairs" in the bundle of orthonormal Lorentz frames $\mathcal{F}(N)$ and indirectly work out the properties of the spacetime. By using the Einstein equations and some global structures in $\mathcal{F}(N)$ we construct the full spacetime at once. There is no "extension process" where you begin with the exterior Schwarzschild solution and find the maximal analytic extension.

We begin by discussing the Cartan structural equation for the bundle of orthonormal frames of a semi-riemannian manifold. We begin to specialize by studying the restrictions imposed on the structural equation if the manifold is a fiber bundle. We do a further specialization to the case that the manifold is a semi-riemannian submersion. We finally study the case that the semi-riemannian submersion arises due to a group action. The Schwarzschild spacetime is a manifold of this type. By studying the properties of the structural equations we can construct the full Kruskal-Szekeres spacetime.

There are extensive computations in these notes because the methods are not familiar to most physicists or mathematicians. It makes extensive use of Cartan's method of the moving frame beyond what most people use. It is more of an abstract use of Cartan's method than the explicit direct computational approach seen in some relativity textbooks. There are some nice uses of the machinery. For this reason some sections are expository in nature and are not directly related to the main topic. 


\section{Frame Bundles}

We wish to globally study a semi-riemannian manifold [9]. Most of the mathematical framework we will be developing works in both the riemannian case and in the lorentzian case. The riemannian language is more standard and for simplicity I will phrase the discussion as if the manifold was riemannian. Of course when we get to black holes we have to work in the lorentzian framework. The only times we have to be careful is if we have a null vector in a subspace. When we encounter such a case I will be extra careful.

We assume that we have an orientable riemannian manifold. The metric allows us to consider the orthonormal bases at each tangent space $T_{x} N$ for $x \in N$. The collection of all such orthonormal bases gives us a principal fiber bundle $\mathcal{F}(N)$, the bundle of all orthonormal frames. This bundle has structure group $\mathrm{SO}(n)$ where $n=\operatorname{dim} N$. Note that $\operatorname{dim} \mathcal{F}(N)=n+\frac{1}{2} n(n-1)$. One of the most important properties of $\mathcal{F}(N)$ is that it has a canonical global coframing [7]. There are $n$ tautologically defined global 1 -forms ${ }^{2}$ on $\mathcal{F}(N)$ that will be denoted by $\left\{\theta^{\mu}\right\}$. There is the unique Levi-Civita connection on $\mathcal{F}(N)$ that gives $\frac{1}{2} n(n-1)$ globally defined 1-forms $\left\{\omega^{\mu}{ }_{\nu}\right\}$ with $\omega_{\mu \nu}=-\omega_{\nu \mu}$. Together the $\frac{1}{2} n(n+1) 1$-forms $\left\{\theta^{\mu}, \omega_{\rho}^{\nu}\right\}$ gives a global coframing of $\mathcal{F}(N)$. The dual basis of vector fields is denoted by $\left(\boldsymbol{e}_{\mu}, \boldsymbol{e}_{\nu \rho}\right)$. The important observation is that we do not have global coordinates on $\mathcal{F}(N)$ but we have something that is almost as good, a global coframe. The frame bundle is the global structure that we are going to use to study the Schwarzschild spacetime.

The Cartan structural equations for the orthonormal frame bundle $\mathcal{F}(N)$ of a riemannian manifold $N$ are [7]

$$
\begin{aligned}
d \theta^{\mu} & =-\omega_{\mu \nu} \wedge \theta^{\nu} \\
d \omega_{\mu \nu} & =-\omega_{\mu \lambda} \wedge \omega_{\lambda \nu}+\frac{1}{2} R_{\mu \nu \rho \sigma}^{N} \theta^{\rho} \wedge \theta^{\sigma} .
\end{aligned}
$$

Note that these are equations on $\mathcal{F}(N)$ and therefore $R_{\mu \nu \rho \sigma}^{N}$ are globally defined functions on $\mathcal{F}(N)$ with certain equivariance transformation laws under the group action. If you consider a local section $s: U \subset N \rightarrow \mathcal{F}(N)$ then the pullback 1forms $\vartheta^{\mu}=s^{*} \theta^{\mu}$ give a local orthonormal coframe on $U \subset N$, the pullback 1-forms $s^{*} \omega_{\mu \nu}=\gamma_{\mu \nu \rho} \vartheta^{\rho}$ give the connection coefficients $\gamma_{\mu \nu \rho}$ in the local orthonormal

\footnotetext{
${ }^{2}$ These are sometimes called the "soldering forms" in the older mathematical literature and in some of the physics literature.
} 
coframe, and $s^{*} R_{\mu \nu \rho \sigma}^{N}=R_{\mu \nu \rho \sigma}^{N} \circ s$ are the components of the Riemann curvature tensor in the local coframe.

Assume we have a local orthonormal coframe $\vartheta^{\mu}$ on $U \subset N$ with Levi-Civita connection $\Gamma_{\mu \nu}=\gamma_{\mu \nu \rho} \vartheta^{\rho}$. Locally we have a trivialization $U \times \operatorname{SO}(n)$ of $\mathcal{F}(N)$. If $(x, g) \in U \times \mathrm{SO}(n)$ then the coframing of $\mathcal{F}(N)$ may be locally expressed in terms of the trivialization as

$$
\begin{aligned}
\theta^{\mu} & =g^{-1} \vartheta^{\mu}, \\
\omega & =g^{-1} d g+g^{-1} \Gamma g .
\end{aligned}
$$

If $x^{\mu}$ are local coordinates on $U$ then $(x, g)$ parametrize $U \times \mathrm{SO}(N)$ and $d x$ and $g^{-1} d g$ are linearly independent on the frame bundle. We physicists usually work downstairs and we usually think of $\omega_{\mu \nu}$ as $\Gamma_{\mu \nu \rho} d x^{\rho}$. Do not do this in this article. The connection lives upstairs! Some formulas look different because we are working upstairs.

Next we discuss how to think about the covariant derivative. A tensor on the base is viewed upstairs as an ordinary vector valued function $\xi^{A}$ (a column vector) that has special transformation laws. Assume we are at a frame ${ }^{3} q=\left(\boldsymbol{e}_{1}, \ldots \boldsymbol{e}_{n}\right)$ and we act on the frame on the right by a rotation matrix $g$ where we find that $q^{\prime}=q g$ then we want $\xi(q g)=\sigma(g)^{-1} \xi(q)$ where $\sigma$ is a representation of $\mathrm{SO}(n)$. The differential of $\xi$ is a 1 -form and we have to specify how $\xi$ changes both along the fiber and transverse to the fiber. We know that $d \xi$ must be expressible as a linear combination of the coframe $\left(\theta^{\mu}, \omega_{\nu \rho}\right)$. The question is which linear combination. Part of the definition of a connection [7] is that $\omega$ restricted to the vertical tangent space is the left invariant form on the Lie algebra. In local formulas (2.2) we see that $\omega=g^{-1} d g$ when tangent to the fibration (given by $d x=0)$. We know how $\xi$ transforms under the $\mathrm{SO}(n)$ action and thus we conclude that

$$
d \xi=-\dot{\sigma}(\omega) \xi+\xi_{; \mu} \theta^{\mu}
$$

Here $\dot{\sigma}$ is the induced Lie algebra representation. The "horizontal" component of $d \xi$ is denoted by $\xi_{; \mu} \theta^{\mu}$ and it is called the covariant differential.

If a vector field

$$
V=V^{\mu} \boldsymbol{e}_{\mu}+\frac{1}{2} V^{\mu \nu} \boldsymbol{e}_{\mu \nu}
$$

\footnotetext{
${ }^{3}$ Think of a frame as a row vector.
} 
on $\mathcal{F}(N)$ generates an isometry then $\mathscr{L}_{V} \theta^{\nu}=0$. A simple computation shows that $^{4}$

$$
0=-V_{\mu \nu} \theta^{\nu}+\omega_{\mu \nu} V^{\nu}+d V_{\mu}=-V_{\mu \nu} \theta^{\nu}+V_{\mu ; \nu} \theta^{\nu} .
$$

We immediately learn two things

$$
\begin{aligned}
0 & =V_{\mu ; \nu}+V_{\nu ; \mu}, \\
V_{\mu \nu} & =\frac{1}{2}\left(V_{\mu ; \nu}-V_{\nu ; \mu}\right) .
\end{aligned}
$$

The first of the above are Killing's equations. The second one determines the $\boldsymbol{e}_{\mu \nu}$ component of the vector field $V$, see (2.4). Next we observe that $0=$ $d \mathscr{L}_{V} \theta^{\mu}=\mathscr{L}_{V}\left(d \theta^{\mu}\right)$. Using the Cartan structural equation we immediately see that $\left(\mathscr{L}_{V} \omega_{\mu \nu}\right) \wedge \theta^{\nu}=0$. An application of the corollary to Cartan's lemma, see Appendix A, yields that $\mathscr{L}_{V} \omega_{\mu \nu}=0$, i.e., the connection is invariant under the infinitesimal isometry.

We will be copiously using the differential forms version of the Frobenius Theorem [10]. To avoid too much terminology we state the theorem in the following practical way.

Theorem 2.1 (Frobenius). Assume that on a manifold $M$ we have a collection of $k$ linearly independent non-vanishing 1 -forms $\left\{\varphi^{\alpha}\right\}$ with the property that $d \varphi^{\alpha}=$ $\xi^{\alpha}{ }_{\beta} \wedge \varphi^{\beta}$ for some 1 -forms $\xi^{\alpha}{ }_{\beta}$. Then through every $q \in M$ there exists a unique maximal connected submanifold $S_{q}$ containing $q$ with $\operatorname{dim} S_{q}=\operatorname{dim} M-k$ such that for every vector field $X$ tangent to $S_{q}$ we have that $\varphi^{\alpha}(X)=0$.

The integrability conditions on the differential forms are sometimes written as $" d \varphi^{\alpha}=0 \bmod \varphi "$.

\section{Fibration}

Let $\pi: N \rightarrow M$ be a fiber bundle where $N$ is a riemannian manifold. The fibers are isomorphic to a manifold $F$. If $x \in M$ then the fiber over $x$ will be denoted by $F_{x}$. Vectors tangent to the fiber will be called vertical and vectors orthogonal to the fiber will be called horizontal. If $\operatorname{dim} N=n$ and $\operatorname{dim} M=p$ then the dimension of the fibers is $q=n-p$. The existence of the fibration allows for a reduction of the structure group $\mathrm{SO}(n)$ of $\mathcal{F}(N)$ to $\mathrm{SO}(p) \times \mathrm{SO}(q)$ obtaining a principal sub-bundle $\mathcal{F}_{\text {red }}(N) \subset \mathcal{F}(N)$. If we introduce indices $i, j, k, \ldots$

\footnotetext{
${ }^{4}$ We use the traditional semi-colon notation to denote covariant derivatives.
} 
"associated to M" to run from $1, \ldots, p$ and indices $a, b, c, d, \ldots$ "associated to the fibers" to run from $p+1, \ldots, n$ then the first structural equation may be written as

$$
\begin{aligned}
& d \theta^{i}=-\omega_{i j} \wedge \theta^{j}-\omega_{i a} \wedge \theta^{a}, \\
& d \theta^{a}=-\omega_{a b} \wedge \theta^{b}-\omega_{a i} \wedge \theta^{i} .
\end{aligned}
$$

Once the structure group is reduced we have that the $\omega_{a i}$ become torsion:

$$
\omega_{a i}=K_{a b i} \theta^{b}-M_{i j a} \theta^{j} .
$$

This requires some explanation and is best understood by looking at local expression (2.2). Once we are on $\mathcal{F}_{\text {red }}(N) \subset \mathcal{F}(N)$ we can no longer move along the group directions that are not tangent to $\mathcal{F}_{\text {red }}(N)$. This means that $g^{-1} d g$ restricted to $\mathcal{F}_{\text {red }}$ vanishes in Lie algebra directions orthogonal to $\mathfrak{s o}(p) \oplus \mathfrak{s o}(q) \subset$ $\mathfrak{s o}(n)$. Thus when restricted to $\mathcal{F}_{\text {red }}(N)$ we only get the $\Gamma$ part of $\omega$ in $(2.2)$. Schematically we have that $\Gamma=\gamma \vartheta=\gamma g \theta$ and this is how (3.2) arises. With this in mind we see that the first of (3.1) becomes

$$
d \theta^{i}=-\omega_{i j} \wedge \theta^{j}-M_{i j a} \theta^{j} \wedge \theta^{a}-\frac{1}{2}\left(K_{a b i}-K_{b a i}\right) \theta^{a} \wedge \theta^{b} .
$$

The pfaffian equations $\theta^{i}=0$ determine an integrable vertical distribution that defines the fibration and therefore the Frobenius theorem requires

$$
K_{a b i}=K_{b a i} .
$$

This is the statement that the second fundamental form for the submanifolds associated with the fibration is symmetric. It is worthwhile to consider the symmetric and anti-symmetric parts of $M$ :

$$
\begin{aligned}
S_{i j a} & =\frac{1}{2}\left(M_{i j a}+M_{j i a}\right), \\
A_{i j a} & =\frac{1}{2}\left(M_{i j a}-M_{j i a}\right) .
\end{aligned}
$$

The structure equation may be written as

$$
d \theta^{i}=-\omega_{i j} \wedge \theta^{j}+A_{i j a} \theta^{a} \wedge \theta^{j}-S_{i j a} \theta^{j} \wedge \theta^{a} .
$$

Following Cartan we try to absorb as much torsion as possible by defining a new connection

$$
\pi_{i j}=\omega_{i j}-A_{i j a} \theta^{a}
$$

The structural equation becomes

$$
d \theta^{i}=-\pi_{i j} \wedge \theta^{j}-S_{i j a} \theta^{j} \wedge \theta^{a} .
$$


Similarly, the second of (3.1) becomes

$$
d \theta^{a}=-\omega_{a b} \wedge \theta^{b}-K_{a b i} \theta^{b} \wedge \theta^{i}-A_{i j a} \theta^{i} \wedge \theta^{j} .
$$

The vanishing of the tensor $A_{i j a}$ is the integrability condition for the distribution defined by $\theta^{a}=0$. You cannot absorb the torsion in $d \theta^{a}$ because $K_{a b i}$ is symmetric under $a \leftrightarrow b$.

Finally we make the following useful remark. If $X$ is a horizontal vector field ,i.e., $\iota_{X} \theta^{a}=0$ then

$$
\mathscr{L}_{X}\left(\theta^{a} \otimes \theta^{a}\right)=2 X^{i} K_{a b i} \theta^{a} \otimes \theta^{b}-2 X^{i} A_{i j a}\left(\theta^{j} \otimes \theta^{a}+\theta^{a} \otimes \theta^{j}\right) .
$$

If the horizontal distribution is integrable then $A_{i j a}=0$ and the equation above simplifies to

$$
\mathscr{L}_{X}\left(\theta^{a} \otimes \theta^{a}\right)=2 X^{i} K_{a b i} \theta^{a} \otimes \theta^{b} .
$$

If $\eta_{F}$ is the volume element on the fiber then

$$
\mathscr{L}_{X} \eta_{F}=X^{i} K^{a}{ }_{a i} \eta_{F} .
$$

SUMMARY: The structural equations for a fibration are

$$
\begin{aligned}
K_{a b i} & =K_{b a i}, \\
M_{i j a} & =S_{i j a}+A_{i j a}, \text { see }(3.5), \\
\omega_{a i} & =K_{a b i} \theta^{b}-M_{i j a} \theta^{j}, \\
\pi_{i j} & =\omega_{i j}-A_{i j a} \theta^{a}, \\
d \theta^{i} & =-\pi_{i j} \wedge \theta^{j}-S_{i j a} \theta^{j} \wedge \theta^{a}, \\
d \theta^{a} & =-\omega_{a b} \wedge \theta^{b}-K_{a b i} \theta^{b} \wedge \theta^{i}-A_{i j a} \theta^{i} \wedge \theta^{j} .
\end{aligned}
$$

3.1. Local Description. Assume our total space $N$ is euclidean space $\mathbb{E}^{n}$ and that after a rotation we locally describe the fibers near the origin as the level sets of the $p$ functions

$$
f^{i}(x)=x^{i}+\frac{1}{2} h_{a b i} x^{a} x^{b}+O\left(x^{3}\right),
$$

where we use cartesian coordinates $\left(x^{i}, x^{a}\right)$. We know that $D_{e_{a}}^{N} e_{i}=e_{b} K_{a b i}+$ $e_{j} \omega^{j}{ }_{i}\left(e_{a}\right)$. Taking the gradient of the function that defines the level sets we see that the normals (to leading order) are given by

$$
e_{i}=\frac{\partial}{\partial x^{i}}+h_{a b i} x^{b} \frac{\partial}{\partial x^{a}} .
$$


Comparing with the connection definition of the extrinsic curvatures we see that at the origin we have that $K_{a b i}=h_{a b i}$. Notice that $K_{a b i}$ is a first order invariant of the metric, i.e., $K \sim \partial g$, while the curvature is second order $R \sim \partial^{2} g$.

For the special case $K_{a b i}=\delta_{a b} S_{i}$ we see that locally our embedded $p$-surface looks like a surface of revolution about the normal $S$ direction.

3.2. Totally Geodesic Fibration. Let's forget the frame bundle and work on the base manifold $N$. The extrinsic curvature tensor (second fundamental forms) is defined by $K(V, W, X)=\left(V, D_{W}^{N} X\right)$ where $V, W$ are vertical vectors and $X$ is a horizontal vector. In a local frame we have $K_{a b i}=\left(e_{a}, D_{e_{b}}^{N} e_{i}\right)$. A geodesic with tangent vector field $V$ that is tangent to a fiber will satisfy the geodesic equation $D_{V}^{N} V=0$ and tangentiality condition $(V, X)=0$ for all horizontal vectors $X$. Since the connection $D^{N}$ is metric compatible we have $0=D_{V}^{N}(V, X)=\left(D_{V}^{N} V, X\right)+\left(V, D_{V}^{N} X\right)=K(V, V, X)$ for all possible $V$. This implies that the second fundamental form must vanish if all geodesics are tangent to the fibration.

3.3. Gauss and Codazzi Equations. The integrability of the vertical distribution allows us to consistently substitute $\theta^{i}=0$ into the equations above by restricting to $F_{x}$, the fiber over $x \in M$.

$$
\begin{aligned}
K_{a b i} & =K_{b a i}, \\
M_{i j a} & =S_{i j a}+A_{i j a}, \text { see }(3.5), \\
\omega_{a i} & =K_{a b i} \theta^{b}, \\
\pi_{i j} & =\omega_{i j}-A_{i j a} \theta^{a}, \\
d \theta^{a} & =-\omega_{a b} \wedge \theta^{b} .
\end{aligned}
$$

Note that

$$
\left.d \theta^{a}\right|_{F_{x}}=-\left.\omega_{a b} \wedge \theta^{b}\right|_{F_{x}}
$$

which tells us that $\left.\omega_{a b}\right|_{F_{x}}$ is the torsion free riemannian connection on $F_{x}$. To work out the curvature we observe that

$$
\frac{1}{2} R_{a b \mu \nu}^{N} \theta^{\mu} \wedge \theta^{\nu}=d \omega_{a b}+\omega_{a c} \wedge \omega_{c b}-\omega_{a i} \wedge \omega_{b i}
$$

Restricting to $F_{x}$ we get the Gauss equation

$$
\frac{1}{2} R_{a b c d}^{N} \theta^{c} \wedge \theta^{d}=\frac{1}{2} R_{a b c d}^{F_{x}} \theta^{c} \wedge \theta^{d}-\frac{1}{2}\left(K_{a c i} K_{b d i}-K_{a d i} K_{b c i}\right) \theta^{c} \wedge \theta^{d} .
$$


This is often written

$$
R_{a b c d}^{N}=R_{a b c d}^{F_{x}}-\left(K_{a c i} K_{b d i}-K_{a d i} K_{b c i}\right) .
$$

Next we derive the Codazzi equation. Note that $\omega_{a i}=K_{a b i} \theta^{b}$ when restricted to $F_{x}$. We have

$$
\begin{aligned}
\frac{1}{2} R_{a i c d}^{N} \theta^{c} \wedge \theta^{d} & =\left.\frac{1}{2} R_{a i \mu \nu}^{N} \theta^{\mu} \wedge \theta^{\mu}\right|_{F_{x}} \\
& =\left.\left(d \omega_{a i}+\omega_{a b} \wedge \omega_{b i}+\omega_{a j} \wedge \omega_{j i}\right)\right|_{F_{x}}, \\
& =\left.\left(d \omega_{a i}+\omega_{a b} \wedge \omega_{b i}+\omega_{a j} \wedge \pi_{j i}\right)\right|_{F_{x}}+\omega_{a j} \wedge A_{j i d} \theta^{d}, \\
& =\left.D\left(K_{a d i} \theta^{d}\right)\right|_{F_{x}}+K_{a c j} A_{j i d} \theta^{c} \wedge \theta^{d} .
\end{aligned}
$$

In the above $D$ is the covariant differential with connection $\left(\omega_{a b}, \pi_{i j}\right)$. If we write $D K_{a b i}=K_{a b i ; j} \theta^{j}+K_{a b i ; c} \theta^{c}$ then the last line of the above may be written as

$$
K_{a d i ; c} \theta^{c} \wedge \theta^{d}+K_{a c j} A_{j i d} \theta^{c} \wedge \theta^{d} .
$$

We have derived the Codazzi equation

$$
R_{a i c d}^{N}=\left(K_{a d i ; c}-K_{a c i ; d}\right)+\left(K_{a c j} A_{j i d}-K_{a d j} A_{j i c}\right) .
$$

\section{Riemannian Submersion}

Many of the spacetimes studied by physicists are semi-riemannian submersions. A submersion $\pi: N \rightarrow M$ of riemannian manifolds is called a riemannian submersion if $d \pi$ preserves the inner product of vectors orthogonal to the fibers [8]. A tangent vector is horizontal if it is orthogonal to the fibers. A riemannian submersion implies a very specific form for the metric. If $x^{i}$ are local coordinates on the base $M$ and if $y^{a}$ are local coordinates on the fiber $F$ then $(x, y)$ are local coordinates on $N$. The fibers are the submanifolds with $x$ fixed. The metric of a submersion is locally of the form

$$
d s_{N}^{2}=g_{i j}(x) d x^{i} d x^{i}+g_{a b}(x, y)\left(d y^{a}+C^{a}{ }_{i}(x, y) d x^{i}\right)\left(d y^{b}+C^{b}{ }_{j}(x, y) d x^{j}\right) .
$$

Sometimes in the physics literature this is referred to as a metric of KaluzaKlein type. If we fix $x$ then the metric on a fiber $g_{a b}(x, y) d y^{a} d y^{b}$ varies as we move along the base. In general curves of constant $x$ will not be orthogonal to curves of constant $y$. On the other hand we have that $\partial / \partial y^{a}$ is orthogonal to the horizonal vector field $\partial / \partial x^{i}-C^{a}{ }_{i} \partial / \partial y^{a}$. The metric on the horizontal space is $g_{i j}(x)$ which is the metric on the base and is independent of choice of 
$y$. O'Neill studied the properties of riemannian submersions and discovered that the geometry was governed by two tensor fields. One tensor field is the second fundamental form (the extrinsic curvature) of the embedding of the fibers in $N$ and the other tensor field is the integrability tensor for the horizontal spaces. We present here a formulation that is equivalent to O'Neill's except that everything is expressed in terms of orthonormal frames adapted to the fibration.

We first do some local analysis. Locally pull back the $\theta$ s from $\mathcal{F}_{\text {red }}(N)$ to $N$ via a section. If $V$ is a vertical vector field on $N$, i.e., tangential to the fibration $\pi: N \rightarrow M$, the condition of a riemannian submersion can be written as $\mathscr{L}_{V}\left(\theta^{i} \otimes \theta^{i}\right)=0$. This is simply the statement that $\theta^{i} \otimes \theta^{i}$ descends to the base. A vertical vector field satisfies $\iota_{V} \theta^{i}=0$. A simple computation shows that

$$
\begin{aligned}
\mathscr{L}_{V}\left(\theta^{i} \otimes \theta^{i}\right) & =-\left(\pi_{i j}(V)+\pi_{j i}(V)\right) \theta^{i} \otimes \theta^{j}+2 V^{a} S_{i j a} \theta^{i} \otimes \theta^{j}, \\
& =2 V^{a} S_{i j a} \theta^{i} \otimes \theta^{j} .
\end{aligned}
$$

The degenerate quadratic form $\theta^{i} \otimes \theta^{i}$ on $N$ descends to a positive definite quadratic form on the base $M$ if

$$
S_{i j a}=0
$$

We can do the same analysis on $\mathcal{F}_{\text {red }}(N)$ but the equations look different. The vector field in this case is a vector field on $\mathcal{F}_{\text {red }}(N)$ and will be of the form

$$
V=V^{a} \boldsymbol{e}_{a}+\frac{1}{2} V^{a b} \boldsymbol{e}_{a b}+\frac{1}{2} V^{i j} \boldsymbol{e}_{i j}
$$

Here $\left(\boldsymbol{e}_{a}, \boldsymbol{e}_{i}, \boldsymbol{e}_{a b}, \boldsymbol{e}_{i j}\right)$ is the basis dual to $\left(\theta^{a}, \theta^{i}, \omega_{a b}, \pi_{i j}\right)$. We want $\mathscr{L}_{V} \theta^{i}=0$ and a brief computation leads to the equation $0=-V_{i j} \theta^{j}+S_{i j b} V^{b} \theta^{j}$. From this we learn that $V_{i j}=0$ and $S_{i j a}=0$. Note that the vector field

$$
V=V^{a} \boldsymbol{e}_{a}+\frac{1}{2} V^{a b} \boldsymbol{e}_{a b}
$$

is of the type that is associated intrinsically with the fibers of the fibration $\pi$ : $N \rightarrow M$. Finally we note that the conditions arising from $\mathscr{L}_{V} \theta^{i}=0$ at $z \in N$ do not depend on the derivatives of the components of $V$ and therefore depend only on $V(z)$ and not its extension to a neighborhood of $z$. 
The structural equations for a riemannian submersion are

$$
\begin{aligned}
K_{a b i} & =K_{b a i} \quad \text { and } \quad A_{i j a}=-A_{j i a}, \\
\omega_{a i} & =K_{a b i} \theta^{b}-A_{i j a} \theta^{j}, \\
\pi_{i j} & =\omega_{i j}-A_{i j a} \theta^{a}, \\
d \theta^{i} & =-\pi_{i j} \wedge \theta^{j}, \\
d \theta^{a} & =-\omega_{a b} \wedge \theta^{b}-K_{a b i} \theta^{b} \wedge \theta^{i}-A_{i j a} \theta^{i} \wedge \theta^{j} .
\end{aligned}
$$

As we mentioned before $K_{a b i}$ are the second fundamental forms (extrinsic curvatures) of the fibration. The tensor $A_{i j a}$ measures the integrability of the horizontal tangent spaces. We use the basic relation that if $\xi$ is a 1 -form then $d \xi(X, Y)=X(\xi(Y))-Y(\xi(X))-\xi([X, Y])$. First we take a section and we pullback the structural equation. We have that $\boldsymbol{e}_{i}$ is a basis for the horizontal spaces of the submersion. If the horizontal spaces are to be integrable then the bracket of two horizontal vector fields must be horizontal. We compute the vertical component of the bracket as follows:

$$
\theta^{a}\left(\left[\boldsymbol{e}_{i}, \boldsymbol{e}_{j}\right]\right)=\boldsymbol{e}_{i}\left(\theta^{a}\left(\boldsymbol{e}_{j}\right)\right)+\boldsymbol{e}_{j}\left(\theta^{a}\left(\boldsymbol{e}_{i}\right)\right)-\left(d \theta^{a}\right)\left(\boldsymbol{e}_{i}, \boldsymbol{e}_{j}\right)=2 A_{i j a} .
$$

Thus we see that the horizontal distribution is integrable if and only if the integrability tensor $A_{i j a}$ vanishes.

We note that $d^{2} \theta^{i}=0$ and therefore

$$
\Pi_{i j} \wedge \theta^{j}=0
$$

where

$$
\Pi_{i j}=d \pi_{i j}+\pi_{i k} \wedge \pi_{k j} .
$$

Wedging (4.3) with $\theta^{k_{1}} \wedge \cdots \wedge \theta^{k_{p-1}}$ we conclude that $\Pi_{i j} \equiv 0 \bmod \theta^{k}$. So we can write $\Pi_{i j}=\Psi_{i j k} \wedge \theta^{k}$ for some 1-forms $\Psi_{i j k}$ that are skew in the indices $i \leftrightarrow j$. From (4.3) we see that $\Psi_{i j k} \wedge \theta^{j} \wedge \theta^{k}=0$. This tells us that $\left(\Psi_{i j k}-\Psi_{i k j}\right) \equiv 0$ $\bmod \theta^{l}$. Since $\Psi_{i j k}$ is symmetric in $j \leftrightarrow k$ modulo $\theta^{l}$ but is it skew in $i \leftrightarrow j$ we conclude that $\Psi_{i j k} \equiv 0 \bmod \theta^{l}$. This tells us that $\Psi_{i j k}=P_{i j k l} \theta^{l}$. Putting this all together we conclude that

$$
\Pi_{i j}=d \pi_{i j}+\pi_{i k} \wedge \pi_{k j}=\frac{1}{2} R_{i j k l}^{M} \theta^{k} \wedge \theta^{l} .
$$

Note that the right hand side is horizontal! This would not be true if we had used the $\omega_{i j}$ connection. 
In the same way we see that the structural equation for $\omega_{a b}$ is given by

$$
\begin{aligned}
d \omega_{a b}= & -\omega_{a c} \wedge \omega_{c b}+\frac{1}{2} R^{F_{x}}{ }_{a b c d} \theta^{c} \wedge \theta^{d} \\
& +\left(R^{N}{ }_{a b c j}-A_{i j b} K_{a c i}+A_{i j a} K_{b c i}\right) \theta^{c} \wedge \theta^{j} \\
& +\frac{1}{2}\left(R^{N}{ }_{a b j k}+A_{i j a} A_{i k b}-A_{i k a} A_{i j b}\right) \theta^{j} \wedge \theta^{k}
\end{aligned}
$$

where $R^{F_{x}}$ is given by the Gauss equation (3.14).

Next we compute the riemannian curvature of the base $M$ by using the riemannian data on the bundle $N$. Note that

$$
\frac{1}{2} R^{N}{ }_{i j \mu \nu} \theta^{\mu} \wedge \theta^{\nu}=d \omega_{i j}+\omega_{i k} \wedge \omega_{k j}-\omega_{a i} \wedge \omega_{a j} .
$$

Substituting the appropriate expressions we find

$$
R^{N}{ }_{i j k l}=R^{M}{ }_{i j k l}+A_{i l a} A_{j k}{ }^{a}-A_{i k a} A_{j l}{ }^{a}-2 A_{i j a} A_{k l}{ }^{a} .
$$

We explicitly state the submersion curvature relations in Table 1 using the O'Neill notation [9] where $\{n\}$ denotes the number of base indices. If you take $a i-\{3\}$ and use the Bianchi identities $\mathrm{B}-\{3\}$ you get $i j-\{3\}$. The term $\mathrm{B}-\{2\}$ is not skew under $a \leftrightarrow b$. From the fact that $R^{N}{ }_{a b j k}+R^{N}{ }_{b a j k}=0$ we learn that

$$
A_{j k a ; b}+A_{j k b ; a}=-K_{a b j ; k}+K_{a b k ; j} .
$$

This relationship is also necessary to ensure that $R^{N}$ aibj $=R^{N}{ }_{b j a i}$. If you insert this relationship into $\mathrm{B}-\{2\}$ you obtain $i j-\{2\}$. Finally we observe that using the above relationship we can write a manifestly symmetric expression for $R^{N}$ aibj. We note that by (4.8), the term between parentheses in the expression $R^{N}{ }_{a i b j}=$ $A_{i k b} A_{j}{ }^{k}{ }_{a}-K_{a c i} K_{b}{ }^{c}{ }_{j}-\left(A_{i j a ; b}+K_{a b i ; j}\right)$ is symmetric under the interchange $a i \leftrightarrow b j$. Therefore we have

$$
R^{N}{ }_{a i b j}=A_{i k b} A_{j}{ }^{k}-K_{a c i} K_{b j}^{c}-\frac{1}{2}\left(A_{i j a ; b}+A_{j i b ; a}+K_{a b i ; j}+K_{b a j ; i}\right) .
$$

Using these relations the structural equation (4.6) may be rewritten as

$$
\begin{aligned}
d \omega_{a b}= & -\omega_{a c} \wedge \omega_{c b}+\frac{1}{2} R^{F_{x}}{ }_{a b c d} \theta^{c} \wedge \theta^{d} \\
& +\left(-K_{c a j ; b}+K_{c b j ; a}\right) \theta^{c} \wedge \theta^{j} \\
& +\frac{1}{2}\left(-A_{j k a ; b}+A_{j k b ; a}-K_{a c j} K_{b}{ }^{c}{ }_{k}+K_{b c j} K_{a}{ }^{c}{ }_{k}\right) \theta^{j} \wedge \theta^{k}
\end{aligned}
$$




\begin{tabular}{|c|c|c|}
\hline plane & $\{n\}$ & Curvature \\
\hline$a b$ & $\{0\}$ & $R_{a b c d}^{N}=R_{a b c d}^{F_{x}}-\left(K_{a c i} K_{b d}^{i}-K_{a d i} K_{b c}^{i}\right)$ \\
\hline$a i$ or $\mathrm{B}$ & $\{1\}$ & $R^{N}{ }_{a i b c}=-K_{a b i ; c}+K_{a c i ; b}-A_{k i b} K_{a c}^{k}+A_{k i c} K_{a b}^{k}$ \\
\hline$a i$ & $\{2\}$ & $R_{a i b j}^{N}=A_{i k b} A_{j}{ }_{a}{ }_{a}-A_{i j a ; b}-K_{a b i ; j}-K_{a c i} K_{b}^{c}{ }_{j}$ \\
\hline$a i$ & $\{3\}$ & $R^{N}{ }_{a i j k}=A_{i j a ; k}-A_{i k a ; j}-2 A_{j k b} K_{a}{ }^{b}{ }_{i}$ \\
\hline$i j$ & $\{2\}$ & $R^{N}{ }_{i j a b}=A_{i k b} A_{j}{ }_{a}{ }_{a}-A_{i k a} A_{j}{ }^{k}{ }_{b}-A_{i j a ; b}+A_{i j b ; a}+K_{a c j} K_{b}{ }^{c}{ }_{i}-K_{a c i} K_{b}{ }^{c}{ }_{j}$ \\
\hline$i j$ & $\{3\}$ & $R^{N}{ }_{i j k b}=A_{i j b ; k}-A_{j k a} K_{b i}^{a}+A_{i k a} K_{b j}^{a}+A_{i j a} K_{b k}^{a} \quad$ [dual Codazzi eq. $]$ \\
\hline$i j$ & $\{4\}$ & $R^{N}{ }_{i j k l}=R_{i j k l}^{M}+A_{i l a} A_{j k}^{a}-A_{i k a} A_{j l}^{a}-2 A_{i j a} A_{k l}^{a}$ \\
\hline $\mathrm{B}$ & $\{2\}$ & $\begin{aligned} R_{a b j k}^{N}= & -A_{i j a} A_{k b}^{i}+A_{i j b} A_{k a}^{i}-2 A_{j k a ; b} \\
& -K_{a b j ; k}+K_{a b k ; j}-K_{b c k} K_{a j}^{c}+K_{b c j} K_{a k}^{c}\end{aligned}$ \\
\hline $\mathrm{B}$ & $\{3\}$ & $\begin{aligned} 0= & -A_{j k a ; l}-A_{k l a ; j}-A_{l j a ; k} \\
& +A_{j k b} K_{a}{ }^{b}{ }_{l}+A_{k l b} K_{a}{ }^{b}{ }_{j}+A_{l j b} K_{a k}{ }^{b}\end{aligned}$ \\
\hline
\end{tabular}

TABLE 1. Relationship of the bundle curvature to the base geometry and fiber geometry for a riemannian submersion. Equations associated with a rotation in the $\mu \nu$-plane, i.e., a consequence of $d \omega_{\mu \nu}$, are labeled by the first column. The second column uses O'Neill's notation where $\{n\}$ denotes the number of horizontal indices. Rows identified with a "B" are equations that are a direct consequence of the Bianchi identities that follow from $d^{2} \theta^{a}=0$.

If we now use the structure equation above look for Bianchi identities in $d^{2} \theta^{a}=0$ we find (4.8) and $\mathrm{B}-\{3\}$ as the identities in addition to the cyclic identity that $R^{F_{x}}$ satisfies. 
SUMMARY: The full structural equations for a riemannian submersion are

$$
\begin{aligned}
K_{a b i}= & K_{b a i} \quad \text { and } \quad A_{i j a}=-A_{j i a}, \\
\omega_{a i}= & K_{a b i} \theta^{b}-A_{i j a} \theta^{j} \\
\pi_{i j}= & \omega_{i j}-A_{i j a} \theta^{a} \\
d \theta^{i}= & -\pi_{i j} \wedge \theta^{j} \\
d \theta^{a}= & -\omega_{a b} \wedge \theta^{b}-K_{a b i} \theta^{b} \wedge \theta^{i}-A_{i j a} \theta^{i} \wedge \theta^{j}, \\
d \pi_{i j}= & -\pi_{i k} \wedge \pi_{k j}+\frac{1}{2} R^{M}{ }_{i j k l} \theta^{k} \wedge \theta^{l}, \\
d \omega_{a b}= & -\omega_{a c} \wedge \omega_{c b}+\frac{1}{2} R^{F_{x}}{ }_{a b c d} \theta^{c} \wedge \theta^{d} \\
& +\left(-K_{c a j ; b}+K_{c b j ; a}\right) \theta^{c} \wedge \theta^{j} \\
& +\frac{1}{2}\left(-A_{j k a ; b}+A_{j k b ; a}-K_{a c j} K_{b}^{c}{ }_{k}+K_{b c j} K_{a k}{ }^{c}\right) \theta^{j} \wedge \theta^{k}
\end{aligned}
$$

If $X, Y$ are horizontal vectors then the sectional curvature is given by

$$
\operatorname{sect}^{N}(X, Y)=\operatorname{sect}^{M}(X, Y)-3 \frac{\left(A_{i k a} X^{i} Y^{k}\right)\left(A_{j l}{ }^{a} X^{j} Y^{l}\right)}{(X, X)(Y, Y)-(X, Y)^{2}} .
$$

A consequence of the above is O'Neill's result [8] that in a strictly riemannian submersion the sectional curvature of the base is "increased" because the second summand subtracts a manifestly positive semi-definite expression. Note that if $N$ is flat then the base always has positive sectional curvature.

4.1. The Ricci Tensor and Ricci Scalar. It is straightforward to write down the Ricci tensor in the case of a submersion:

$$
\begin{aligned}
& R_{b d}^{N}=R_{b d}^{F_{x}}-K_{b d ; i}{ }^{i}-K_{c i}^{c} K_{b d}{ }^{i}+A^{i k}{ }_{b} A_{i k d}, \\
& R^{N}{ }_{a i}=-K_{b i ; a}^{b}+K^{b}{ }_{a i ; b}+A^{j}{ }_{i a ; j}-A_{i j b} K_{a b}{ }^{j}+A_{k i a} K_{b}{ }^{b k}, \\
& R^{N}{ }_{i j}=R^{M}{ }_{i j}-2 A_{i k d} A_{j}{ }^{k d}-K_{c d i} K^{c d}{ }_{j}-\frac{1}{2}\left(K^{a}{ }_{a i ; j}+K^{a}{ }_{a j ; i}\right) .
\end{aligned}
$$

The Ricci scalar is easily seen to be

$$
R^{N}=R^{M}+R^{F_{x}}-2 K_{a}^{a i}{ }_{; i}-K_{a b i} K^{a b i}-K^{c}{ }_{c i} K_{a}{ }^{a i}-A_{i j a} A^{i j a} .
$$

\section{The Group Action}

5.1. Transitive Case and Invariant Tensors. Assume we have a connected Lie group $G$ acting transitively on a connected manifold $M$ via isometries. Let 
$H \subset G$ be the isotropy group at $x \in M$. We know that $M \approx G / H$ and that $\pi: G \rightarrow M$ is a principal $H$-bundle. We assume that the Lie algebra is a reductive Lie algebra: $\mathfrak{g}=\mathfrak{m} \oplus \mathfrak{h}$ with $[\mathfrak{h}, \mathfrak{m}] \subset \mathfrak{m}$. There is a canonical identification of $T_{x} M$ with $\mathfrak{m}$. It is well known that $G$-invariant tensors on $M \approx G / H$ are in a 1-1 correspondence with $H$-invariant tensors on $\mathfrak{m}$, see [9]. The argument is roughly as follows. Assume $S$ is a $G$-invariant tensor on $M$ then if $y=g \cdot x$ then $S_{y}=g \cdot S_{x}$. If $y=g^{\prime} \cdot x$ then $g^{\prime}=g h$ for some $h \in H$. We immediately see that since $g \cdot S_{x}=g^{\prime} \cdot S_{x}$ we must have that $h \cdot S_{x}=S_{x}$ for all $h \in H$.

5.2. General Case. Assume we have a connected Lie group $G$ acting on $N$ via isometries. The orbit of $z \in N$ by the $G$ action will be denoted by $\mathcal{O}_{z}$. If $G_{z}$ is the isotropy group at $z$ then $\mathcal{O}_{z} \approx G / G_{z}$. We assume all the $G_{z} \subset G$ are isomorphic as we vary $z \in N$. The orbits will foliate $N$. Under our assumptions, the dimensions of the orbits are constant and we have a fibration $\pi: N \rightarrow M$ such that if $\pi(z)=x$ then the fiber at $x$ is isomorphic to the orbit $F_{x} \approx \mathcal{O}_{z}$.

Let $\operatorname{dim} \mathcal{O}_{z}=q$ then the foliation reduces the structure group of the orthonormal frame bundle from $\mathrm{SO}(n)$ to $\mathrm{SO}(p) \times \mathrm{SO}(q)$. Let $y \in \mathcal{O}_{z}$ such that $y=g \cdot z$ for $g \in G$. If we write $T_{z} N=T_{z} \mathcal{O}_{z} \oplus T_{z} \mathcal{O}_{z}^{\perp}$ and $T_{y} N=T_{y} \mathcal{O}_{z} \oplus T_{y} \mathcal{O}_{z}^{\perp}$ then we have that $g \in G$ takes $T_{z} \mathcal{O}_{z}$ isometrically to $T_{y} \mathcal{O}_{z}$ and $T_{z} \mathcal{O}_{z}^{\perp}$ isometrically to $T_{y} \mathcal{O}_{z}^{\perp}$. The isometric action on the normal bundle to the orbits tells us that we have a riemannian submersion and therefore structural equations (4.2) are valid. Additionally we have an isometric action on the fibers. The Killing vector field is tangential to the orbits therefore its lift to the reduced frame bundle is of the form

$$
V=V^{a} \boldsymbol{e}_{a}+\frac{1}{2} V_{a b} \boldsymbol{e}_{a b}+\frac{1}{2} V_{i j} \boldsymbol{e}_{i j}
$$

Using the invariance conditions $\mathscr{L}_{V} \theta^{i}=0, \mathscr{L}_{V} \theta^{a}=0$, and structural equations (4.2) leads to

$$
\begin{aligned}
V_{i j} & =0, \\
V_{a ; b}+V_{b ; a} & =0, \\
V_{a b}-V_{a ; b} & =0, \\
V_{a ; i}-K_{a b i} V^{b} & =0,
\end{aligned}
$$


where $D V_{a}=d V_{a}+\omega_{a b} V_{b}=V_{a ; b} \theta^{b}+V_{a ; i} \theta^{i}$. The first equation tells us that the $G$ action on $T_{z} \mathcal{O}_{z}^{\perp}$ is trivial ${ }^{5}$. The middle two equations tell us that when we restrict to the orbit $\mathcal{O}_{z}$ then we have the familiar Killing equations. If the orbit bends, i.e., the second fundamental form is nonvanishing, then the last equation tell us that the normal derivative of the Killing vector field is nontrivial and is determined by the second fundamental form.

At $z \in N$ we have that $G_{z}$ acts as an isometry on $T_{z} N \approx T_{z} \mathcal{O}_{z} \oplus T_{z} \mathcal{O}_{z}^{\perp}$. Since $\mathcal{O}_{z}$ is the orbit of $G$, the isotropy subgroup $G_{z}$ acts as an isometry and leaves invariant the tangent space $T_{z} \mathcal{O}_{z}$. Consequently, $G_{z}$ also acts as an isometry on the orthogonal complement $T_{z} \mathcal{O}_{z}^{\perp}$. The action of $G$ is transitive on the orbit $\mathcal{O}_{z}$. If we look at the structural equations (4.2) we see that the second fundamental tensor $K_{a b i}(z)$ and the integrability tensor $A_{i j a}(z)$ must be invariant tensors under the $G_{z}$ action by generalizing the arguments given in Section 5.1 to the normal bundle $T_{z} \mathcal{O}_{z}^{\perp}$. Also remember $K_{a b i}$ and $A_{i j a}$ are ordinary functions on the reduced frame bundle. The structure equations show that these functions are constants under the action of $G$. To see this consider a Killing vector $V$ then we note that $\mathscr{L}_{V}\left(d \theta^{a}\right)=d\left(\mathscr{L}_{V} \theta^{a}\right)=0$ and therefore

$$
0=-\left(\mathscr{L}_{V} \omega_{a b}\right) \wedge \theta^{b}-V\left(K_{a b i}\right) \theta^{b} \wedge \theta^{i}-V\left(A_{i j a}\right) \theta^{i} \wedge \theta^{j}
$$

Note there is a unique term that is a form of degree 2 in the horizontal direction and therefore $V\left(A_{a b i}\right)=0$ and we conclude that $A_{i j a}$ is constant under the action of $G$. This reduces the equation to $\left[\mathscr{L}_{V} \omega_{a b}-V\left(K_{a b i}\right) \theta^{i}\right] \wedge \theta^{b}=0$. Cartan's lemma tells us that

$$
\mathscr{L}_{V} \omega_{a b}-V\left(K_{a b i}\right) \theta^{i}=B_{a b c} \theta^{c},
$$

where $B_{a b c}=B_{a c b}$. Symmetrizing the displayed equation under $a \leftrightarrow b$ we see that $V\left(K_{a b i}\right) \theta^{i}=\frac{1}{2}\left(B_{a b c}+B_{b a c}\right) \theta^{c}$. We immediately see that $V\left(K_{a b i}\right)=0$ and $B_{a b c}=-B_{b a c}$. This tells us that $K_{a b i}$ is constant under the action of $G$. Also $B_{a b c}$ is skew under $a \leftrightarrow b$ but symmetric under $b \leftrightarrow c$ and therefore $B_{a b c}=0$ and consequently we also learn as expected $\mathscr{L}_{V} \omega_{a b}=0$. The same type of statements will be true for the curvatures. We summarize below.

\footnotetext{
${ }^{5}$ This is one of these left-right action confusions. The reader is urged to understand this in the $S^{2} \approx \mathrm{SO}(3) / \mathrm{SO}(2)$ example.
} 
Proposition 5.1. Let $u \in \mathcal{F}_{\text {red }}(N)$ and let $B_{u} \subset \mathcal{F}_{\text {red }}(N)$ be the orbit ${ }^{6}$ of $u$ under the action of $G$. The functions $K_{a b i}, A_{i j a}$ and the curvatures are constant on $B_{u}$.

We have some information of the derivatives of various tensors. As an example we consider the case of the extrinsic curvature. Remember that $K_{a b i}$ are functions on $\mathcal{F}_{\text {red }}(N)$ and the differential $d K_{a b i}$ in the various directions on $\mathcal{F}_{\text {red }}(N)$ are given by

$$
d K_{a b i}=-\omega_{a c} K_{c b i}-\omega_{b c} K_{a c i}-\pi_{i j} K_{a b j}+K_{a b i ; j} \theta^{j}+K_{a b i ; c} \theta^{c} .
$$

If we differentiate along the direction of the Killing vector field we find using (5.2) and the previous equation that

$$
0=-V_{a c} K_{c b i}-V_{b c} K_{a c i}+K_{a b i ; c} V^{c} .
$$

If the integrability tensor $A_{i j a}$ vanishes then the horizontal distribution is integrable. Each leaf of the foliation is isometric to the base $M$ and also each leaf is orthogonal to the fibers. This can easily happen because of the group action. Assume that under the $G_{z}$ action there are no fixed vectors in $T_{z} \mathcal{O}_{z}$. The integrability tensor $A_{i j}{ }^{a}(z)$ is an invariant tensor under $G_{z}$ that transforms just like a vector in the vector space $T_{z} \mathcal{O}_{z}$ and thus it must vanish. This extends everywhere because we are assuming that the $G$ action leads to a bona fide fibration and the vector spaces $T_{z} \mathcal{O}_{z}$, groups $G_{z}$ and the associated representations are all isomorphic. This leads to the following proposition.

Proposition 5.2. If under the $G_{z}$ action there are no fixed vectors in $T_{z} \mathcal{O}_{z}$ then $A_{i j a}=0$. The horizontal distribution is integrable and its integral submanifolds are orthogonal to the fibers.

These methods are also useful for studying axisymmetric solutions. For example, Theorem 7.1.1 in Wald's book [6] can be proven by using the methods discussed above.

5.3. The Basic Example. The basic non-trivial example is given by the $\mathrm{SO}(3)$ action on $N=\mathbb{E}^{3} \backslash\{\boldsymbol{0}\}$. The fibration $\pi: N \rightarrow M$ has fibers isomorphic to $S^{2}$ and the base is $M=\mathbb{R}_{+}$. We can easily write down the structural equations (4.2) by noting that since $\operatorname{dim} M=1$ the integrability tensor $A_{i j a}$ vanishes identically.

\footnotetext{
${ }^{6}$ It can be shown that $B_{u}$ is a sub-bundle of $\mathcal{F}_{\text {red }}(N)$.
} 
Note that the indices $i, j=1$ and they will be suppressed. At $z \in N$, the isotropy group is isomorphic to $\mathrm{SO}(2)$ and therefore the second fundamental form must of the form $K_{a b}=k \delta_{a b}$ where $k$ is constant on each $S^{2}$ fiber and can only depend on the radial direction, i.e., it is the pullback of a function on $M$. Let's write the 1forms as $(\rho, \theta, \phi)$ where we are using a notation analogous to spherical coordinates $(r, \vartheta, \varphi)$.

$$
\begin{aligned}
\omega_{a i} & =k \theta^{a}, \\
d \theta & =-\omega \wedge \phi-k \theta \wedge \rho, \\
d \phi & =+\omega \wedge \theta-k \phi \wedge \rho, \\
d \rho & =0 .
\end{aligned}
$$

Next we observe that since $\mathbb{E}^{3}$ is flat we have that $d \omega_{a i}+\omega_{a b} \wedge \omega_{b i}+\omega_{a j} \wedge \omega_{j i}=0$. This greatly simplifies to $d\left(k \theta^{a}\right)+k \omega \wedge \theta^{a}=0$. A little algebra yields $\left(d k+k^{2} \rho\right) \wedge$ $\theta^{a}=0$ and consequently

$$
d k=-k^{2} \rho
$$

This immediately tells us that $k$ is constant on the fibers as expected. From $d^{2} \theta=d^{2} \phi=0$ we learn that $d \omega=A \theta \wedge \phi$. Note that $\omega$ is invariant on an orbit, the area element $\theta \wedge \phi$ is invariant on the orbit, therefore $A$ must be constant on the orbits. The equation $d^{2} \omega=0$ then tells us that $d A+2 k A \rho=0$.

Since $d \rho=0$ we can set $\rho=d r$ for some function $r$ specified up to an additive constant. We can easily integrate (5.5) to obtain $1 / k=r+c$. By redefining the coordinate $r$ we can set $c=0$. Thus we find that the second fundamental form is determined by

$$
k=\frac{1}{r}
$$

The curvature is given by

$$
d \omega=\frac{A_{0}}{r^{2}} \theta \wedge \phi
$$

where $A_{0} \in \mathbb{R}$. $A_{0}$ may be determined by topological or by geometrical considerations. 
In summary the structural equations associated with the $\mathrm{SO}(3)$ action on $\mathbb{E}^{3} \backslash\{\mathbf{0}\}$ become

$$
\begin{aligned}
\rho & =d r, \\
d \theta & =-\omega \wedge \phi-\frac{1}{r} \theta \wedge d r, \\
d \phi & =+\omega \wedge \theta-\frac{1}{r} \phi \wedge d r, \\
d \omega & =\frac{A_{0}}{r^{2}} \theta \wedge \phi .
\end{aligned}
$$

On a level surface of constant $r$ we have that $(\theta, \phi, \omega)$ are the Maurer-Cartan forms for $\mathrm{SO}(3)$. The constant $A_{0}$ may be determined via topological arguments or coordinate arguments where you find that $A_{0}=1$.

The space $N=\mathbb{E}^{3} \backslash\{\mathbf{0}\}$ is flat and the geodesics are straight lines. It is not a complete riemannian manifold because radial geodesics reach the origin in finite affine parameter. It is clear that by adding an extra point $N \cup\{\mathbf{0}\} \approx \mathbb{E}^{3}$ becomes a complete riemannian manifold. We can try to do the same analysis by thinking of $N$ in terms of its structural equation (5.8). This analysis is much more complicated. The structural equations are non-singular as long as $r \neq 0$ and this is true in $N$. Analyzing the structural equation you can see that radial geodesics get to $r=0$ in finite time and thus it appears that there is a hole in the space. You have to work little hard with the structural equations to show that the apparent singularity at $r=0$ is removable and that by adding a point at $r=0$ we get the complete smooth manifold $\mathbb{E}^{3}$.

\section{Spherically Symmetric $(3+1)$ Geometry}

Assume $N$ is a 4-dimensional lorentzian manifold that is both orientable and time orientable. This means that the structure group of of the orthonormal Lorentz frame bundle is $\mathrm{SO}^{\uparrow}(1,3)$, the connected component of the Lorentz group. We assume there is an $\mathrm{SO}(3)$ action that leaves the metric invariant and that the orbit of a point is a 2-dimensional spacelike surface. Let $\mathcal{O}_{p}$ be the orbit through $p \in N$. This action leads to a foliation of $N$ by the 2-dimensional orbits. Under some assumptions of a constant dimensionality of the orbits we can assume that this foliation is actually a fibration. Our hypothesis tells us that $\operatorname{dim} \mathcal{O}_{p}=2$. If $G_{p}$ is the isotropy group at $p$ then $\operatorname{dim} G_{p}=1$. This tells us that $\mathcal{O}_{p} \approx \mathrm{SO}(3) / G_{p} \approx S^{2}$. The $\mathrm{SO}(3)$ action identifies a spacelike 2-plane 
$T_{p} \mathcal{O}_{p} \subset T_{p} N$ at each $p \in N$ that induces a reduction of the structure group of the lorentzian orthonormal frame bundle from $\mathrm{SO}^{\uparrow}(1,3)$ to $\mathrm{SO}^{\uparrow}(1,1) \times \mathrm{SO}(2)$. A consequence is that there is only one $\omega_{a b}$ and one $\pi_{i j}$. If we use some type of "Schwarzschild spherical coordinates" denoted by $(t, r, \vartheta, \phi)$. Then we will have non-vanishing connections $\omega_{\vartheta \phi}$ and $\pi_{t r}$. If $\pi: N \rightarrow M$ is our fiber bundle and if $\pi(p)=x$ then the fiber over $x$ is given by $F_{x}=\mathcal{O}_{p}$.

The general discussion of Section 5.2 tells us that we have a pseudo-riemannian submersion. At $p \in N$ we can write $T_{p} N=T_{p} \mathcal{O}_{p} \oplus T_{p} \mathcal{O}_{p}^{\perp}$ and the $\operatorname{SO}(3)$ action tells us that both the riemannian metric on $T_{p} \mathcal{O}_{p}$ and the lorentzian metric on $T_{p} \mathcal{O}_{p}^{\perp}$ are invariant under the $\mathrm{SO}(3)$ action. At $p \in N$, all geometrical structures must be invariant under the isotropy group action $G_{p} \approx \mathrm{SO}(2)$. The action of $G_{p}$ on $T_{p} \mathcal{O}_{p}$ is the standard $\mathrm{SO}(2)$ action and the action on $T_{p} \mathcal{O}_{p}^{\perp}$ is automatically trivial because there is no $\mathrm{SO}(2)$ subgroup in $\mathrm{SO}^{\uparrow}(1,1)$. Because of this we can conclude that the integrability tensor $A_{i j a}$ for the horizontal spaces of the submersion must vanish. At $p \in N$ the integrability tensor may be viewed as a map $A_{p}: \Lambda^{2}\left(T_{p} \mathcal{O}_{p}^{\perp}\right) \rightarrow T_{p} \mathcal{O}_{p}$. We note that $\Lambda^{2}\left(T_{p} \mathcal{O}_{p}^{\perp}\right)$ is one dimensional so $A_{p}$ on the normalized area element gives a preferred vector on $T_{p} \mathcal{O}_{p}$. Said differently we must have $A_{i j a}=\epsilon_{i j} v_{a}$. A non-vanishing vector field $v$ tangential to $\mathcal{O}_{p}$ is not $G_{p}$ invariant and must therefore vanish ${ }^{7}$. This tells us that $v$ must vanish. Thus we have learned that the horizontal subspaces are integrable.

Similarly the second fundamental forms $K_{a b i}(p)$ must be invariant under $G_{p}$ otherwise the structure group will be further reduced. This immediately tells us that $K_{a b i}=\delta_{a b} S_{i}$ where $\sigma=S_{i} \theta^{i}$ will be called the second fundamental 1-form.

We can extend this argument and conclude that $R_{i a}^{N}=0$ and $R_{a b}^{N} \propto \delta_{a b}$. We will denote by $\eta=\left(\begin{array}{cc}-1 & 0 \\ 0 & +1\end{array}\right)$ the Minkowski metric on $T \mathcal{O}_{p}^{\perp}$.

The structural equations (4.2) applied to this case become

$$
\begin{aligned}
\omega_{a i} & =S_{i} \theta^{a}, \\
\pi_{i j} & =\omega_{i j}, \\
d \theta^{i} & =-\pi_{i j} \wedge \theta^{j}, \\
d \theta^{a} & =-\omega_{a b} \wedge \theta^{b}-S_{i} \theta^{a} \wedge \theta^{i} .
\end{aligned}
$$

\footnotetext{
${ }^{7}$ The tensor $A_{i j a}$ must be invariant under $G_{p}$ if not then the structure group will be further reduced.
} 
Next we explore additional properties that follow from the $\mathrm{SO}(3)$ action. First we observe that $\omega_{a b}$ did not get modified by the symmetry breakdown therefore we know that under the action of the $\mathrm{SO}(3)$ Killing vector

$$
V=V^{a} \boldsymbol{e}_{a}+\frac{1}{2} V_{a ; b} \boldsymbol{e}_{a b},
$$

the connection is invariant $\mathscr{L}_{V} \omega_{a b}=0$. Also $\mathscr{L}_{V} \omega_{a i}=0$ because $\omega_{a i}$ in (6.1) comes from restriction to the reduced orthonormal frame bundle $\mathcal{F}_{\text {red }}(N)$ and the Killing vector field $V$ is tangential to $\mathcal{F}_{\text {red }}(N) \subset \mathcal{F}(N)$. This immediately implies that $\iota_{V} d S_{i}=S_{i ; a} V^{a}=0$ from which we conclude that $S_{i ; a}=0$. Next we show that the 1 -form $\sigma=S_{i} \theta^{i}$ is invariant under the $\mathrm{SO}(3)$ action. We have that $\mathscr{L}_{V} \sigma=\iota_{V} d \sigma+d \iota_{V} \sigma$. We observe that $d \sigma=S_{i ; j} \theta^{j}+S_{i ; a} \theta^{a}=S_{i ; j} \theta^{j}$. Since $\iota_{V} \sigma=0$ and $\iota_{V} d \sigma=0$ we see that $\mathscr{L}_{V} \sigma=0$.

Alternatively, this can also be seen by looking at the reduced structure equations (6.1) directly. First we observe that $\mathscr{L}_{V} \sigma=\mathscr{L}_{V}\left(S_{i} \theta^{i}\right)=\left(\iota_{V} d S_{i}\right) \theta^{i}=$ $V^{a} S_{i ; a} \theta^{i}$. Next we note that $0=d\left(\mathscr{L}_{V} \theta^{a}\right)=\mathscr{L}_{V}\left(d \theta^{a}\right)$ from which we conclude that $0=\left(\mathscr{L}_{V} \omega_{a b}\right) \wedge \theta^{b}+\left(\mathscr{L}_{V} \sigma\right) \wedge \theta^{a}$. If we write $\omega_{a b}=\epsilon_{a b} \omega$ we see that the previous equation becomes

$$
\begin{aligned}
&\left(\mathscr{L}_{V} \omega\right) \wedge \theta^{3}+V^{a} S_{i ; a} \theta^{i} \wedge \theta^{2}=0, \\
&-\left(\mathscr{L}_{V} \omega\right) \wedge \theta^{2}+V^{a} S_{i ; a} \theta^{i} \wedge \theta^{3}=0 .
\end{aligned}
$$

By inspection we see that the unique solution is $\mathscr{L}_{V} \omega=0$ and $S_{i ; a}=0$. In conclusion we have that

$$
\begin{aligned}
d \sigma & =-\frac{1}{2}\left(S_{i ; j}-S_{j ; i}\right) \theta^{i} \wedge \theta^{j}, \\
S_{i ; a} & =0 .
\end{aligned}
$$

Notice that (4.8) tells us that $S_{i ; j}=S_{j ; i}$ and therefore we learn that $d \sigma=0$. This is also a consequence of

$$
0=d^{2} \theta^{a}=-\left(\epsilon_{a b} \omega+\delta_{a b} d \sigma\right) \wedge \theta^{b} .
$$

One of the equations above is $\omega \wedge \theta^{3}+d \sigma \wedge \theta^{2}=0$. If we wedge with $\theta^{3}$ we find that $d \sigma \wedge \theta^{2} \wedge \theta^{3}=0$. If we use (6.3) we immediately learn that $S_{0 ; 1}=S_{1 ; 0}$, i.e., $\sigma$ is a closed 1-form: $d \sigma=0$. Therefore we see that $d \omega \wedge \theta^{a}=0$. Using a similar argument we see that

$$
d \omega=k^{F_{x}} \theta^{2} \wedge \theta^{3} .
$$

We will shortly return to this equation. 
Using the submersion curvature results in Table 1 we immediately see that

$$
\begin{aligned}
R_{i j k l}^{N} & =R_{i j k l}^{M}, \\
R_{i j a b}^{N} & =0, \\
R_{i j k a}^{N} & =0 . \\
R_{a i b j}^{N} & =-\delta_{a b}\left(S_{i ; j}+S_{i} S_{j}\right) .
\end{aligned}
$$

Note that the $G_{p}$ action implies that $R_{a b c i}^{N}=R_{c i a b}^{N}=0$ and this can explicitly be verified from the formulas. We point out that

$$
R_{i j k l}^{M}=k^{M} \epsilon_{i j} \epsilon_{k l}=-k^{M}\left(\eta_{i k} \eta_{j l}-\eta_{i l} \eta_{j k}\right),
$$

because $M$ is two dimensional. The negative sign in the last equality is due to the negative sign in the Minkowski metric.

We observe that since the fiber $\mathcal{O}_{p}$ is 2-dimensional we have that $R_{a b c d}^{F_{x}}=$ $k^{F_{x}} \epsilon_{a b} \epsilon_{c d}=k^{F_{x}}\left(\delta_{a c} \delta_{b d}-\delta_{a d} \delta_{b c}\right)$. Again using the results from Table 1 we see that

$$
R^{N}{ }_{a b c d}=\left(k^{F_{x}}-S^{i} S_{i}\right)\left(\delta_{a c} \delta_{b d}-\delta_{a d} \delta_{b c}\right) .
$$

Putting all this together we learn that the Cartan structural equation for the $\mathrm{SO}(2)$ curvature (4.10) may be written in this case as

$$
d \omega_{a b}=k^{F_{x}} \theta^{a} \wedge \theta^{b} .
$$

The SO(3)-orbits are "round" 2-spheres and we have that

$$
k^{F_{x}}=\frac{1}{r^{2}} .
$$

Here $r: \mathcal{F}_{\text {red }}(N) \rightarrow \mathbb{R}_{+}$is the radius of the 2 -sphere. Since know that $k^{F_{x}}$ is constant on each orbit there exists a globally defined function $r_{M}: M \rightarrow \mathbb{R}_{+}$such that $r$ is the pullback to the bundle of $r_{M}$. The function $r_{M}$ is just the radius of the fibering $S^{2}$. We will avoid all the notation required and simply refer to the radius function as $r$ and implicitly assume its domain on context. Note that $d k^{F_{x}}$ is independent of $\theta^{a}$ because of the $\mathrm{SO}(3)$ action and it is also independent of the connections because it is invariant under $\mathrm{SO}^{\uparrow}(1,1) \times \mathrm{SO}(2)$. Computing $0=d^{2} \omega_{a b}$ we find

$$
0=\left(d k^{F_{x}}+2 k^{F_{x}} \sigma\right) \wedge \theta^{2} \wedge \theta^{3} .
$$

Since $k^{F_{x}}$ and $\sigma$ are $\mathrm{SO}(3)$ invariant we learn that

$$
d k^{F_{x}}+2 k^{F_{x}} \sigma=0 .
$$


Using (6.12) we see that

$$
\sigma=\frac{1}{r} d r=d(\log r) .
$$

We have learned that $\sigma$ is exact as will be collaborated by an independent argument later. In fact, the above equation will be valid everywhere if the fibration is non-singular. Next we observe that if $d r=r_{i} \theta^{i}$ then

$$
S_{i}=\frac{r_{i}}{r}
$$

The Gauss equation (6.10) becomes

$$
R^{N}{ }_{a b c d}=\left(\frac{1-\|d r\|_{M}^{2}}{r^{2}}\right)\left(\delta_{a c} \delta_{b d}-\delta_{a d} \delta_{b c}\right)
$$

The Ricci tensor is computed using (4.13). First we note that as expected by $G_{p}$ invariance we have $R_{a i}^{N}=0$. Doing the computations we find

$$
\begin{aligned}
R_{i j}^{N} & =-2\left(S_{i ; j}+S_{i} S_{j}\right)-k^{M} \eta_{i j}, \\
& =-2 \frac{r_{i ; j}}{r}-k^{M} \eta_{i j}, \\
R_{a b}^{N} & =\left[k^{F}-\left(S_{; i}^{i}+2 S^{i} S_{i}\right)\right] \delta_{a b} \\
& =\left(\frac{1-\|d r\|_{M}^{2}-r r^{i} ;}{r^{2}}\right) \delta_{a b}, \\
& =\left(\frac{2-\square\left(r^{2}\right)}{2 r^{2}}\right) \delta_{a b} .
\end{aligned}
$$

The wave operator is defined by $\square f=\eta^{i j} f_{; i ; j}$ where each semi-colon denotes a covariant derivative.

The Cartan structural equations associated with the $\mathrm{SO}(3)$ action on $N$ are

$$
\begin{aligned}
d \theta^{0} & =+\pi \wedge \theta^{1}, \\
d \theta^{1} & =+\pi \wedge \theta^{0}, \\
d \pi & =k^{M} \theta^{0} \wedge \theta^{1}, \quad \text { where } \pi=\pi_{01}, \\
d \theta^{2} & =-\omega \wedge \theta^{3}-\frac{1}{r} \theta^{2} \wedge d r, \\
d \theta^{3} & =+\omega \wedge \theta^{2}-\frac{1}{r} \theta^{3} \wedge d r, \\
d \omega & =+\frac{1}{r^{2}} \theta^{2} \wedge \theta^{3}, \quad \text { where } \quad \omega=\omega_{23} .
\end{aligned}
$$


The equation $d^{2} \pi=0$ tells you that $d k^{M}=k_{1}^{M} \theta^{1}+k_{2}^{M} \theta^{2}$, i.e., $k^{M}$ is the pullback to the frame bundle of a function on $M$. The geometry is determined by two functions, $r$ and $k^{M}$, that are the pullbacks of functions on $M$. If $r$ and $k^{M}$ are non-singular in a neighborhood of a point $q \in \mathcal{F}_{\text {red }}(N)$ then the structural equations can be integrated to locally construct the frame bundle.

If $r$ and $k^{M}$ are independent functions in a neighborhood in $M$, i.e., $d r \wedge d k^{M} \neq$ 0 , then the inverse function theorem tells you that $\varphi: p \in M \mapsto\left(r(p), k^{M}(p)\right) \in$ $\mathbb{R}^{2}$ can be used as a local coordinate system for the neighborhood.

The converse of the above will be important to us later. If $r$ and $k^{M}$ are dependent functions in a neighborhood in $M$, i.e., $d r \wedge d k^{M}=0$, then $k^{M}$ is a function of $r$. The reason is that $d\left(k^{M} d r\right)=0$ and therefore locally there exists a function $F$ such that $d F=k^{M} d r$.

\section{Vacuum Einstein Equations}

The vacuum Einstein equations are $R_{i j}^{N}=0$ and $R_{a b}^{N}=0$. Using (6.17) these may be written as

$$
\begin{aligned}
-2 \frac{r_{i ; j}}{r}-k^{M} \eta_{i j} & =0 \\
\left(\frac{1-\|d r\|_{M}^{2}-r r_{; i}^{i}}{r^{2}}\right) \delta_{a b} & =0 .
\end{aligned}
$$

Taking the trace of each of the equations above we learn

$$
\begin{aligned}
\frac{r_{; i}^{i}}{r}+k^{M} & =0, \\
\frac{r_{; i}^{i}}{r}-\frac{1-\|d r\|_{M}^{2}}{r^{2}} & =0 .
\end{aligned}
$$

Taking the difference of the equations above we see that

$$
k^{M}=-\frac{1}{r^{2}}\left(1-\|d r\|_{M}^{2}\right) .
$$

The dalembertian term of (7.2) may be rewritten as

$$
\square\left(r^{2}\right)=2,
$$

a hyperbolic equation for $r^{2}$. In some sense, the area of the fibering 2-sphere, $A=4 \pi r^{2}$, is a propagating field on $M$ with a constant source. 
Finally we point out that automatically there is an extra killing vector. Consider a "horizontal" vector field

$$
X=X^{i} \boldsymbol{e}_{i}+\frac{1}{2} X^{i j} \boldsymbol{e}_{i j}
$$

then we have

$$
\begin{aligned}
\mathscr{L}_{X} \theta^{a} & =\frac{X^{i} r_{i}}{r} \theta^{a}, \\
\mathscr{L}_{X} \theta^{i} & =-X_{i j} \theta^{j}+D X^{i} .
\end{aligned}
$$

Notice that for any horizontal $X$, the change in $\theta^{a} \otimes \theta^{a}$ is conformal ${ }^{8}$. On the other hand if $X^{i} \propto \epsilon^{i j} r_{j}$ then the first equation above is automatically zero. We will see that we can make the second also zero. Choose $X^{i}=\epsilon^{i k} r_{k} F(r)$ for some real valued function of $r$. We note that $D X_{i}=X_{i ; j} \theta^{j}+X_{i ; a} \theta^{a}$. The Killing conditions require $X_{i ; a}=0$, i.e., $X$ is intrinsically associated with the base $M$. The second displayed equation above also requires $X_{i ; j}+X_{j ; i}=0$. Next we note that

$$
\begin{aligned}
X_{i ; j} & =\epsilon_{i}{ }^{k} r_{k ; j} F(r)+\epsilon_{i}{ }^{k} r_{k} r_{j} F^{\prime}(r), \\
& =\epsilon_{i k} r^{k} r_{j} F^{\prime}(r)-\frac{1}{2} \epsilon_{i j} k^{M} r F(r) .
\end{aligned}
$$

The condition for the flow to generate an isometry is $F^{\prime}(r)=0$ or equivalently $F(r)=F_{0}$ where $F_{0}$ is a constant. In conclusion we have an additional Killing vector given by

$$
X^{i}=-\epsilon^{i j} r_{j}
$$

The vectors $r^{i}$ and $X^{i}$ are Minkowski orthogonal, $r_{i} X^{i}=0$, and that

$$
\|X\|_{M}^{2}=-\|\nabla r\|_{M}^{2} .
$$

Note that if $\nabla r$ is spacelike then $X$ is timelike and vice-versa. If $\nabla r$ is lightlike then $X$ is also lightlike and vice-versa.

Next we observe that the Lie derivative of the metric on the fibers along the direction of $\nabla r$ is given by

$$
\mathscr{L}_{\nabla r}\left(\theta^{a} \otimes \theta^{a}\right)=2 \frac{\|\nabla r\|_{M}^{2}}{r} \theta^{a} \otimes \theta^{a} .
$$

\footnotetext{
${ }^{8}$ This has to be true because there is a unique round metric on $S^{2}$ up to scale. The horizontal vector field moves you to another point where the associated fiber is also a round $S^{2}$.
} 
We conclude that if $\nabla r$ is space-like then the area of the 2-sphere increases in the direction of $\nabla r$. If $\nabla r$ is time-like then the area of the 2 -sphere decreases in the direction of $\nabla r$.

7.1. Properties of the radius function. Next we derive a differential equation satisfied by $\nu=r^{i} r_{i}=\|d r\|_{M}^{2}$.

$$
\begin{aligned}
d \nu & =2 r^{i} r_{i ; j} \theta^{j}, \\
& =-r k^{M} d r, \\
& =\frac{1-\nu}{r} d r .
\end{aligned}
$$

A little algebra leads to the equation

$$
d((\nu-1) r)=0
$$

The solution to this equation is elementary and given by

$$
\nu=\|d r\|_{M}^{2}=1+\frac{c}{r},
$$

where $c \in \mathbb{R}$ is a constant of integration. We also have using (7.7)

$$
\|X\|_{M}^{2}=-\|d r\|_{M}^{2}=-\left(1+\frac{c}{r}\right) .
$$

Using (7.3) we see that

$$
k^{M}=\frac{c}{r^{3}} .
$$

Next we determine the constant $c$. Here we need to make a physical assumption. We assume that in the spacetime $N$ there is a region that is asymptotically minkowskian and looks like the gravitational far field of a localized mass distribution. The Cartan structural equations tell us that as $r \rightarrow+\infty$ our geometry becomes asymptotically minkowskian. The equation for geodesic deviation says that $D_{u} D_{u} \eta=D_{u} D_{\eta} u=\left[D_{u}, D_{\eta}\right] u=R^{N}(u, \eta) u$. In the instantaneous rest frame we have $u=\boldsymbol{e}_{t}$ and we look at $\eta=\eta^{r} \boldsymbol{e}_{r}$. Our relative radial acceleration equation becomes

$$
\frac{d^{2} \eta^{r}}{d t^{2}}=R_{t t r}^{r} \eta^{r}=R_{r t t r} \eta^{r}=-k^{M} \eta^{r}=-\frac{c}{r^{3}} \eta^{r} .
$$

Newtonian mechanics tells us that $\ddot{r}=-M / r^{2}$ where $M$ is the mass of the star. We have that $\eta^{r}=\delta r$ and therefore $\ddot{\eta}^{r}=\left(2 M / r^{3}\right) \eta^{r}$. We immediately see that

$$
c=-R_{S} \quad \text { where } \quad R_{S}=2 M
$$


is called the Schwarzschild radius.

Finally we observe that we can define a closed 1 -form $\tau$ by ${ }^{9}$

$$
\tau=\frac{r_{1} \theta^{0}+r_{0} \theta^{1}}{1-R_{S} / r}=\frac{-\epsilon_{i j} r^{i} \theta^{j}}{1-R_{S} / r}
$$

with the property that $\tau(X)=1$. Note that $\tau$ is not defined if $r=R_{S}$. Up to scale we have that $\tau$ is basically $* d r$, the Hodge dual on $\mathrm{M}$ of $d r$.

It is worthwhile to summarize the data that determines our geometry:

$$
\begin{aligned}
R_{S} & =2 M, \quad \text { Schwarzschild radius, } \\
r_{M} & : M \rightarrow \mathbb{R}_{+}, \quad \text { radius of the fibering } S^{2}, \\
d r & =r_{i} \theta^{i}, \\
r_{i ; j} & =\frac{R_{S}}{2 r^{2}} \eta_{i j}, \\
X^{i} & =-\epsilon^{i j} r_{j}, \\
\|d r\|_{M}^{2} & =1-\frac{R_{S}}{r}, \\
\|X\|_{M}^{2} & =-\left(1-\frac{R_{S}}{r}\right), \\
\tau & =\frac{r_{1} \theta^{0}+r_{0} \theta^{1}}{1-R_{S} / r}=\frac{-\epsilon_{i j} r^{i} \theta^{j}}{1-R_{S} / r} \text { and } d \tau=0, \\
\|\tau\|_{M}^{2} & =-\left(1-\frac{R_{S}}{r}\right)^{-1} .
\end{aligned}
$$

\footnotetext{
${ }^{9}$ On a two dimensional manifold, a locally non-vanishing 1-form $\alpha$ always defines a local foliation because the Frobenius condition $d \alpha \equiv 0 \bmod \alpha$ is automatically satisfied. Furthermore, the Frobenius theorem states that there exists functions $f$ and $g$ such that $\alpha=f d g$. In our case we have that $\alpha=* d r$.
} 
The Cartan structural equations for the reduced frame bundle of the Schwarzschild spacetime are

$$
\begin{aligned}
d \theta^{0} & =+\pi \wedge \theta^{1}, \\
d \theta^{1} & =+\pi \wedge \theta^{0}, \\
d \pi & =-\frac{R_{S}}{r^{3}} \theta^{0} \wedge \theta^{1}, \quad \text { where } \pi=\pi_{01}, \\
d \theta^{2} & =-\omega \wedge \theta^{3}-\frac{1}{r} \theta^{2} \wedge d r, \\
d \theta^{3} & =+\omega \wedge \theta^{2}-\frac{1}{r} \theta^{3} \wedge d r, \\
d \omega & =+\frac{1}{r^{2}} \theta^{2} \wedge \theta^{3}, \quad \text { where } \omega=\omega_{23} .
\end{aligned}
$$

Note that the only singularity in the structural equations occurs where $r=0$. For this reason we expect the frame bundle of the Schwarzschild manifold to be smooth everywhere as long as $r \neq 0$. In particular we do not expect any type of singularity when $r_{M}=R_{S}$. The exceptional properties of the Schwarzschild solution at $r_{M}=R_{S}$ occur because of the behavior of $d r$ at $r=R_{S}$. The only potential problems with the structural equations occur at $r=0$. What type of singularity is at $r=0$ ? Is it removable as in the example of $\mathbb{E}^{3} \backslash\{\mathbf{0}\}$ or is it a true singularity?

We can make a consistency check on equations ${ }^{10}(7.27),(7.28),(7.29)$. If we make a conformal rescaling $\hat{\theta}^{a}=\theta^{a} / r$ then these equations may be written as

$$
\begin{aligned}
& d \hat{\theta}^{2}=-\omega \wedge \hat{\theta}^{3}, \\
& d \hat{\theta}^{3}=+\omega \wedge \hat{\theta}^{2}, \\
& d \omega=+\hat{\theta}^{2} \wedge \hat{\theta}^{3} .
\end{aligned}
$$

These equations are easily identifiable. They are the Cartan structural equations for the orthogonal frame bundle of the unit 2-sphere. Note that they are the Maurer-Cartan equations for the group $\mathrm{SO}(3)$ and thus the frame bundle of $S^{2}$ is isomorphic to $\mathrm{SO}(3)$. The base space for this frame bundle is precisely $\mathrm{SO}(3) / \mathrm{SO}(2) \approx S^{2}$.

7.2. Geodesics. We work out some properties of the geodesics on $M$ by using Cartan's method [11], see Appendix C. It is useful to introduce a null basis

\footnotetext{
${ }^{10}$ This also applies to (5.8).
} 
for the canonical 1-forms on the Lorentz frame bundle of $M$ by defining $\theta^{ \pm}=$ $\theta^{0} \pm \theta^{1}$ then the pullback of the metric on $M$ to the frame bundle is given by $-\frac{1}{2}\left(\theta^{+} \otimes \theta^{-}+\theta^{-} \otimes \theta^{+}\right)$. We also note that

$$
\begin{aligned}
d \theta^{ \pm} & = \pm \pi \wedge \theta^{ \pm}, \\
d \pi & =\frac{R_{S}}{2 r^{3}} \theta^{+} \wedge \theta^{-} .
\end{aligned}
$$

Remember that we are working "upstairs"!

Let $\theta^{i}=\bar{\theta}^{i}+u^{i} d \lambda$ and $\pi=\bar{\pi}$ for $\lambda \geq 0$. Here barred 1-forms are independent of $d \lambda$ analogous to $\vartheta$ and $\varpi$ in Appendix C. The initial conditions are that $\bar{\theta}^{i}(0)=0, \partial_{\lambda} \bar{\theta}^{i}(0)=d u^{i}$, and $\bar{\pi}(0)=0$. Differentiating once we see that

$$
\begin{aligned}
\frac{\partial \bar{\theta}^{ \pm}}{\partial \lambda} & =d u^{ \pm} \mp \bar{\pi} u^{ \pm}, \\
\frac{\partial \bar{\pi}}{\partial \lambda} & =\frac{R_{S}}{2 r^{3}}\left(u^{+} \bar{\theta}^{-}-u^{-} \bar{\theta}^{+}\right) .
\end{aligned}
$$

Differentiating again we see that

$$
\left(\begin{array}{l}
\partial^{2} \bar{\theta}^{+} / \partial \lambda^{2} \\
\partial^{2} \bar{\theta}^{-} / \partial \lambda^{2}
\end{array}\right)=\frac{R_{S}}{2 r^{3}}\left(\begin{array}{cc}
u^{+} u^{-} & -\left(u^{+}\right)^{2} \\
-\left(u^{-}\right)^{2} & u^{+} u^{-}
\end{array}\right)\left(\begin{array}{c}
\theta^{+} \\
\theta^{-}
\end{array}\right)
$$

Next we derive an ODE that $r$ satisfies along a geodesic. We note that $d r / d \lambda=$ $r_{+} u^{+}+r_{-} u^{-}$. Next we remember that $d r_{ \pm}=\mp \pi r_{ \pm}+r_{ \pm ;+} \theta^{+}+r_{ \pm ;-} \theta^{-}$and that $r_{+;+}=r_{-;-}=0$ by (7.18). Therefore along a geodesic we have that

$$
\begin{aligned}
& \frac{d r_{+}}{d \lambda}=-\frac{R_{S}}{4 r^{2}} u^{-}, \\
& \frac{d r_{-}}{d \lambda}=-\frac{R_{S}}{4 r^{2}} u^{+} .
\end{aligned}
$$

We immediately see that

$$
\frac{d^{2} r}{d \lambda^{2}}=\frac{R_{S}}{2 r^{2}}\|u\|_{M}^{2}
$$

The equations that describe the exponential map (7.31) are complicated but the equation that describes the evolution of $r$ along a geodesic (7.33) is relatively simple.

The case of a null radial geodesic is particularly simple because $d^{2} r / d \lambda^{2}=0$. If the horizontal lift of the null geodesic begins at a point $p \in \mathcal{F}(M)$ with $r(p)=r_{p}$ and $d r(p)=r_{i}(p) \theta^{i}(p)$ then the evolution of $r$ along the lift is

$$
r(\lambda)=r_{p}+\lambda\left(r_{+}(p) u^{+}+r_{-}(p) u^{-}\right) .
$$


There are four cases of null geodesics to analyze corresponding to

$$
\left(u^{+}, u^{-}\right) \in\{(+1,0),(0,+1),(-1,0),(0,-1)\} \text {. }
$$

The latter two cases may be considered with the first two by allowing $\lambda$ to be negative. In the first case we have that $r(\lambda)=r_{p}+\lambda r_{+}(p)$, and in the second case we have $r(\lambda)=r_{p}+\lambda r_{-}(p)$. Choose a Lorentz frame $p \in \mathcal{F}(M)$, if $r_{+}(p)>0$ then $r_{+}\left(p^{\prime}\right)>0$ for all $p^{\prime}$ in the same fiber because the action of the $(1+1)$ dimensional Lorentz group translates to an action $r_{ \pm} \rightarrow e^{ \pm \eta} r_{ \pm}$where $\eta$ is the rapidity. This means that we can define the following four open subsets of $M$ :

$$
\begin{aligned}
U_{\mathrm{I}} & =\left\{q \in M \mid r_{+}(p)>0, r_{-}(p)<0\right\}, \\
U_{\mathrm{II}} & =\left\{q \in M \mid r_{+}(p)<0, r_{-}(p)<0\right\}, \\
U_{\mathrm{III}} & =\left\{q \in M \mid r_{+}(p)>0, r_{-}(p)>0\right\}, \\
U_{\mathrm{IV}} & =\left\{q \in M \mid r_{+}(p)<0, r_{-}(p)>0\right\} .
\end{aligned}
$$

In the above $p \in \mathcal{F}(M)$ is any Lorentz orthonormal frame at $q \in M$.

By hypothesis, our space-time manifold $N$ has a region where it is asymptotically like Minkowski space. In such a region a light ray can go radially inward $\left(u^{+}, u^{-}\right)=(0,1)$ or radially outward $\left(u^{+}, u^{-}\right)=(1,0)$. In that asymptotically Minkowski region we can choose a $p \in \mathcal{F}(M)$ with the property that $r_{+}(p)>0$ and $r_{-}(p)<0$ and thus we conclude that $U_{\mathrm{I}} \neq \emptyset$ and that the familiar asymptotic exterior lies in $U_{\mathrm{I}}$. According to (7.34), an inward future directed radial null geodesic will have $r(\lambda)=r_{p}+\lambda r_{-}(p)$. Two important observations are that for finite positive affine parameter the light ray will cross $r=R_{S}$ and in finite affine parameter it will also hit $r=0$. This last observation says that our space may have a singularity because the Cartan structural equations have a singularity at $r=0$. We will not address the question of whether this is a real or a removable singularity. We will concentrate on what happens to null geodesics at $r=R_{S}$.

7.3. Schwarzschild Geometry without Coordinates. The key to understanding the geometry of the Schwarzschild solution is to understand the level sets of the radius function $r: M \rightarrow \mathbb{R}_{+}$. For all practical purposes, both physical and mathematical, we can take $M$ to be simply connected. Topology tells us that $M$ has a universal simply connected cover $\kappa: \widetilde{M} \rightarrow M$. We have a fiber bundle $\pi: N \rightarrow M$. We can use the covering map $\kappa$ to obtain the pull back bundle 
$\tilde{\pi}: \widetilde{N} \rightarrow \widetilde{M}$ and we can pull back all metrics. The conclusion is that we might as well as well assume that $M$ is simply connected. We do this now.

An important ingredient in our discussion is that we can use $r$ as a Morse function $^{11}$ to learn about $M$. The Einstein equation (7.18) tells that that the critical points of $r$ are non-degenerate. Let's briefly review the argument. Assume I have a smooth function $f: X \rightarrow \mathbb{R}$ where $X$ is a manifold. The point $p \in X$ is a critical point if $\left.d f\right|_{p}=0$. The hessian of $f$ at $x_{0}$ can be defined intrinsically but it is easier to do it in terms of local coordinates. Let $\left(x^{i}\right)$ and $\left(y^{i}\right)$ be two local coordinate systems. We observe that the matrix of second derivatives has a non-tensorial transformation law

$$
\frac{\partial^{2} f}{\partial x^{i} \partial x^{j}}=\frac{\partial y^{k}}{\partial x^{i}} \frac{\partial y^{l}}{\partial x^{j}} \frac{\partial^{2} f}{\partial y^{k} \partial y^{l}}+\frac{\partial^{2} y^{k}}{\partial x^{i} \partial x^{j}} \frac{\partial f}{\partial y^{k}},
$$

except at a critical point $p$ where $\partial f / \partial y(p)=0$ and the above reduces to

$$
\left.\frac{\partial^{2} f}{\partial x^{i} \partial x^{j}}\right|_{p}=\left.\left.\left.\frac{\partial y^{k}}{\partial x^{i}}\right|_{p} \frac{\partial y^{l}}{\partial x^{j}}\right|_{p} \frac{\partial^{2} f}{\partial y^{k} \partial y^{l}}\right|_{p} .
$$

The next thing we observe is that the hessian at a critical point is given by the second covariant derivative with respect to any connection $\Gamma$. The reason is that

$$
\left(D_{i} D_{j} f\right)(p)=\frac{\partial^{2} f}{\partial x^{i} \partial x^{j}}(p)-\Gamma^{k}{ }_{i j}(p) \frac{\partial f}{\partial x^{k}}(p)=\frac{\partial^{2} f}{\partial x^{i} \partial x^{j}}(p) .
$$

If $r: M \rightarrow \mathbb{R}_{+}$has critical points then they must be non-degenerate because of (7.18).

Assume the radius function $r: M \rightarrow \mathbb{R}_{+}$has a critical point at $p \in M$. We know by (7.18) that this critical point is non-degenerate. We also know by Morse's lemma [13] that in a neighborhood of $p$ we can find local coordinate $\left(y^{0}, y^{1}\right)$ centered at $p$ that are Minkowski orthonormal at $p$ such that in the neighborhood we have that

$$
r(y)=R_{S}+\frac{-\left(y^{0}\right)^{2}+\left(y^{1}\right)^{2}}{4 R_{S}} .
$$

The neighborhood of any critical point of the function $r_{M}$ looks like Figure 1.

We begin analyzing the properties of $r_{M}: M \rightarrow \mathbb{R}_{+}$. Let $U_{>}=\{p \in$ $\left.M \mid r_{M}(p)>R_{S}\right\}$ and let $U_{<}=\left\{p \in M \mid 0<r_{M}(p)<R_{S}\right\}$. Note that $U_{>}$ and $U_{<}$are both open subsets of $M$. It is clear from (7.20) that $d r_{M}(p) \neq 0$ if

\footnotetext{
${ }^{11}$ There is an application of Morse theory to black holes by Carter [12, p. 187] but it is different from ours.
} 


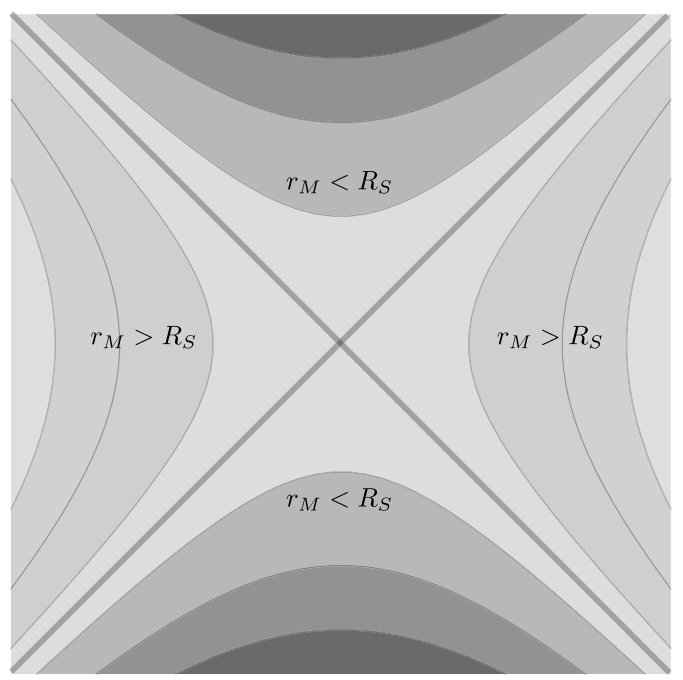

FiguRE 1. Behavior of the function $r_{M}$ near the critical point.

$p \in U_{<} \cup U_{>}$. The implicit function theorem tells us that the level sets of the function $r_{M}$ give a good foliation on $U_{<} \cup U_{>}$. The only question remains what happens at $r_{M}=R_{S}$ where we note that $\|d r\|_{M}^{2}=0$ by (7.20). Since the metric is Minkowski we cannot conclude that $d r=0$, but we do know that if there is a critical point then it must be non-degenerate and that it must have $r_{M}=R_{S}$.

Next we establish that $r$ must have a critical point. We choose a point $p_{1} \in$ $U_{\mathrm{I}} \subset M$ that is in the asymptotic Minkowski region $r_{M} \gg R_{S}$ where $r_{+}\left(q_{1}\right)>0$ and $r_{-}\left(q_{1}\right)<0$ and let $q_{1} \in \mathcal{F}(M)$ be a Lorentz frame at $p_{1}$. We will construct a null broken horizontal curve beginning at $q_{1}$ that takes us to the critical point. Begin with an inward null horizontal curve with initial data $\left(u^{+}, u^{-}\right)=(0,1)$. Inserting into (7.34) we see that $r(\lambda)=r_{q_{1}}+\lambda r_{-}\left(q_{1}\right)$ and therefore the horizontal curve arrives to a point $q_{2} \in \mathcal{F}(M)$ where the sphere radius is the Schwarzschild radius at $\lambda_{R_{S}}=\left(R_{S}-r_{q_{1}}\right) / r_{-}\left(q_{1}\right)>0$. Note that according to $(7.32)$ we have that

$$
\begin{aligned}
\frac{d r_{+}}{d \lambda} & =-\frac{R_{S}}{4\left(r_{q_{1}}+\lambda r_{-}\left(q_{1}\right)\right)^{2}}, \\
\frac{d r_{-}}{d \lambda} & =0 .
\end{aligned}
$$


Thus $r_{-}$is constant along this horizontal curve and we have $r_{-}\left(q_{2}\right)=r_{-}\left(q_{1}\right)<0$. We know that at $r=R_{S}$ we have that $\|d r\|_{M}^{2}=0$ and therefore $r_{+}\left(q_{2}\right)=0$. We can verify this explicitly. Solving the ODE for $r_{+}$we see that

$$
r_{+}(\lambda)=r_{+}\left(q_{1}\right)-\frac{R_{S}}{4 r_{-}\left(q_{1}\right) r_{q_{1}}}+\frac{R_{S}}{4 r_{-}\left(q_{1}\right)\left(r_{q_{1}}+\lambda r_{-}\left(q_{1}\right)\right)} .
$$

Inserting $\lambda=\lambda_{R_{S}}$ and doing some algebra we find the desired result.

At the point $q_{2}$ where $r_{-}\left(q_{2}\right)<0$ and $r_{+}\left(q_{2}\right)=0$ we begin a new horizontal curve with initial velocity $\left(u^{+}, u^{-}\right)=(-1,0)$. Along this curve we have $r(\lambda)=R_{S}$ is constant and

$$
\begin{aligned}
& \frac{d r_{+}}{d \lambda}=0, \\
& \frac{d r_{-}}{d \lambda}=+\frac{1}{4 R_{S}} .
\end{aligned}
$$

Thus $r_{+}=0$ along this curve and $r_{-}(\lambda)=r_{-}\left(q_{2}\right)+\lambda / 4 R_{S}$. Thus in finite positive $\lambda$ we will get to a point $q_{*}$ where $r_{-}\left(q_{*}\right)=0$. This is the critical point of $r$ that we sought.

We have established that if $M$ has an asymptotic Minkowski region then there exists a critical point of the radius function $r_{M}$. Can there be more than one critical point? The answer is no under our hypotheses. The pictorial topological argument is that near each critical point we have a situation that looks like Figure 1. It is very hard to see how two copies of the figure can be put together consistently. You can also give a more analytical argument that has two parts.

The first part is essentially running the proof of the existence backwards. Namely we observe that if we start at a critical point $p_{*} \in M$ of $r_{M}$ then $r_{M}\left(p_{*}\right)=R_{S}$ and there are two null geodesics emanating from $p_{*}$ and along each we have that $r_{M}=R_{S}$, see Figure 1. Lift the geodesics to horizontal curves. Along the first horizontal curve we have that $r_{+}=0$ and $r_{-}$is a strictly monotonic function of the affine parameter, and along the other horizontal curve we have the opposite: $r_{-}=0$ and $r_{+}$is a strictly monotonic function of the affine parameter. This immediately tells us that we cannot have another critical point along the null geodesics emanating from $p_{*}$.

The second part of the argument is a bit more involved. It is proof by contradiction. We develop the intuition by studying the case where we assume that there are nearby timelike separated critical points $p_{*}$ and $p_{*}^{\prime}$ as in Figure 2. We 


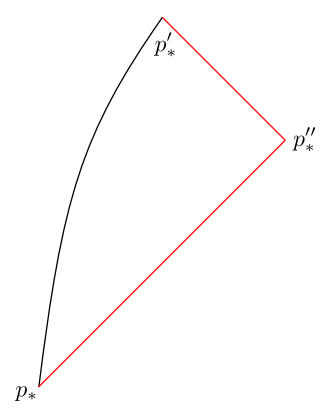

FiguRE 2. Two nearby timelike separated critical points $p_{*}$ and $p_{*}^{\prime}$ connected by a path and also by a broken null geodesic.

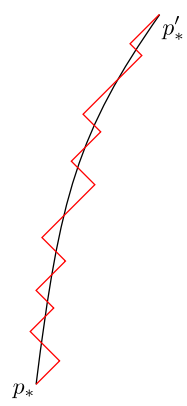

Figure 3 . Two causally separated critical points $p_{*}$ and $p_{*}^{\prime}$ connected by a causal path and also by a broken null geodesic.

begin with a horizontal null curve with initial tangent vector $\left(u^{+}, u^{-}\right)=(1,0)$ at $q_{*} \in \mathcal{F}(M)$ over $p_{*} \in M$. We evolve the curve until it reaches a point $q_{*}^{\prime \prime}$ over $p_{*}^{\prime \prime}$. According to $(7.32)$ we have that $r_{+}\left(q_{*}^{\prime \prime}\right)=0$ and $r_{-}\left(q_{*}^{\prime \prime}\right)<0$. Next we begin a null curve with initial tangent vector $\left(u^{+}, u^{-}\right)=(0,1)$ that will take us to a point $q_{*}^{\prime}$ over the critical point $p_{*}^{\prime}$. But according to $(7.32)$ we have that $r_{-}\left(q_{*}^{\prime}\right)=r_{-}\left(q_{*}^{\prime \prime}\right)<0$. This contradicts $d r\left(q^{\prime}\right)=0$. More generally, assume that you have two critical points on $M$ with $p_{*}^{\prime}$ in the future of $p_{*}$. Since $M$ is connected there is a causal curve between them. We approximate the causal curve by the zig-zag path of null geodesics as in Figure 3. If we apply the previous argument piece by piece to the zig-zag we conclude that $d r\left(q_{*}^{\prime}\right) \neq 0$. The argument can be extended to the case where the conjectured critical points are not causally connected by using broken null geodesics that are future and past directed. 


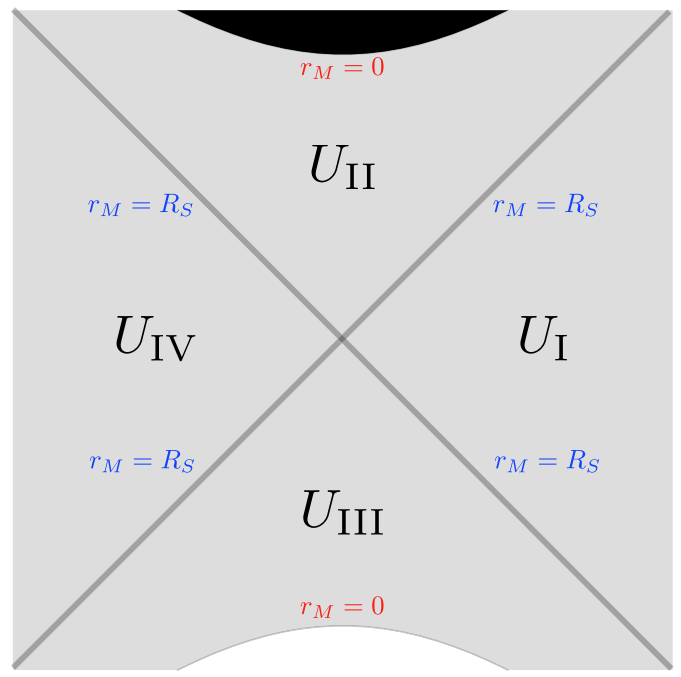

Figure 4. The four regions of the Kruskal spacetime with the black hole and the white hole singularities as indicated.

7.4. The Kruskal Spacetime. Since there is only one critical point we have the standard Kruskal diagram, see Figure 4, of the Schwarzschild geometry with the four regions associated with (7.35). The asymptotic Minkowski region is in Region $U_{\text {I }}$. In Region $U_{\text {II }}$ we have that $r_{ \pm}<0$ and (7.34) tells us that all future directed null geodesics end up at $r_{M}=0$ in finite affine parameter. Therefore light rays cannot escape Region $U_{\text {II }}$. This is the black hole region. Region $U_{\text {III }}$ is the white hole region which is the time reversal image of the black hole region. Region $U_{\text {IV }}$ is the parity image of $U_{\mathrm{I}}$. The two Minkowski like regions are causally disconnected. The properties of the radial null geodesics in the various regions are easily determined using (7.34).

Note that the Killing vector field $X$ is a null vector field on the null lines defined by $r_{M}=R_{S}$ in the Kruskal spacetime. If we think in terms of the fibration $\pi: N \rightarrow M$. The fiber over where the two line intersects is called the the bifurcation 2-sphere. The fibers over the two null geodesics at $r_{M}=R_{S}$ define the bifurcate Killing horizon.

There are real singularities at $r_{M}=0$. We do not get any new insight into the nature of the singularities using these methods. For this reason we will not say anything about it. 
7.5. Schwarzschild Coordinates. We conclude by using our geometrical data to write down the metric in standard Schwarzschild coordinates. On the open set $V \subset \mathcal{F}(M)$ that is the complement to the closed set $r^{-1}\left(R_{S}\right) \subset \mathcal{F}(M)$ we can write

$$
\begin{aligned}
\tau & =\frac{r_{1}}{1-R_{S} / r} \theta^{0}+\frac{r_{0}}{1-R_{S} / r} \theta^{1}, \\
d r & =r_{0} \theta^{0}+r_{1} \theta^{1} .
\end{aligned}
$$

The inverse relationship is

$$
\begin{aligned}
\theta^{0} & =-r_{1} \tau-\frac{r_{0}}{1-R_{S} / r} d r \\
\theta^{1} & =-r_{0} \tau+\frac{r_{1}}{1-R_{S} / r} d r
\end{aligned}
$$

From this we learn that on $V$ we have

$$
-\left(\theta^{0}\right)^{2}+\left(\theta^{1}\right)^{2}=-\left(1-R_{S} / r\right) \tau^{2}+\left(1-R_{S} / r\right)^{-1}(d r)^{2} .
$$

which is the metric in standard Schwarzschild coordinates because $\tau$ is a closed 1-form and therefore locally exact ${ }^{12}, \tau=d t$. By taking a section you can pull these structures back to the base $M$.

7.6. Redshift without Coordinates. This discussion is treated in standard texts. We have a timelike Killing vector field $X$ in regions I and IV and we restrict to observers in either of these regions. Let $k$ be a tangent vector to a null geodesic, i.e., $D_{k} k=0$ and $\|k\|^{2}=0$. It is elementary to show that $D_{k}(X \cdot k)=0$. In other words $X \cdot k$ is constant along the geodesic. We know that if $k$ is the wave vector of a beam of light then an observer at $q \in N$ with (timelike) 4-velocity $u$ will measure the frequency to be $\omega(q)=-u \cdot k$. Consider two observers $E$ and $O$ at fixed radii $r_{E}$ and $r_{O}$. A photon is emitted by $E$ and observed by $O$. We note that $u_{E}=\left(1-R_{S} / r_{E}\right)^{-1 / 2} X_{E}$ and that $u_{O}=\left(1-R_{S} / r_{O}\right)^{-1 / 2} X_{O}$ because the observers are at fixed radii. Using the constancy of $X \cdot k$ along the null geodesic we conclude that

$$
\frac{\omega_{O}}{\omega_{E}}=\sqrt{\frac{1-R_{S} / r_{E}}{1-R_{S} / r_{O}}}
$$

\footnotetext{
${ }^{12}$ There are really four different functions $t_{\mathrm{I}}, t_{\mathrm{II}}, t_{\mathrm{III}}, t_{\mathrm{IV}}$ corresponding to the four regions $U_{\mathrm{I}}, U_{\mathrm{II}}, U_{\mathrm{III}}, U_{\mathrm{IV}}$ that make up $V$.
} 


\section{ACKNOWLEDGMENTS}

I would like to thank G. Galloway for reading an earlier draft of the manuscript. I would also like to thank R. Bryant who explained to me some of the intricacies of the method of moving frames about 15 years ago. Over the years, I.M. Singer has generously explained and shared his geometrical insights about the theory and the importance of principal bundles. I would like to also thank him for reading an earlier draft. This work was supported in part by National Science Foundation grants PHY-0244261 and PHY-0554821.

\section{Appendix A. Cartan's Lemma}

Cartan's lemma is the observation that if $\left\{\varphi^{i}\right\}$ is a linearly independent collection of 1 -forms and if $\left\{\alpha_{i}\right\}$ are 1 -forms such that $\alpha_{i} \wedge \varphi^{i}=0$ then there exists coefficients $\left\{a_{i j}\right\}$ with $a_{i j}=a_{j i}$ such that $\alpha_{i}=a_{i j} \varphi^{j}$.

A corollary to Cartan's lemma is the statement that if you have a collection of 1-forms $\left\{\beta_{i j}\right\}$ with $\beta_{i j}=-\beta_{j i}$ and if $\beta_{i j} \wedge \varphi^{j}=0$ then $\beta_{i j}=0$. To prove this we note that Cartan's lemma implies that there exists coefficients $b_{i j k}=b_{i k j}$ such that $\beta_{i j}=b_{i j k} \varphi^{k}$. But $b_{i j k}$ is skew symmetric under $i \leftrightarrow j$ but symmetric under $j \leftrightarrow k$ and therefore $b_{i j k}=0$. This corollary is responsible for the uniqueness of the Levi-Civita connection, i.e., the fundamental lemma of riemannian geometry. 


$$
\begin{aligned}
& \theta^{ \pm}=\theta^{0} \pm \theta^{1} \\
& \frac{\partial}{\partial \theta^{ \pm}}=\frac{1}{2}\left(\frac{\partial}{\partial \theta^{0}} \pm \frac{\partial}{\partial \theta^{1}}\right) \\
& d s^{2}=-\left(\theta^{0}\right)^{2}+\left(\theta^{1}\right)^{2}, \\
& d s^{2}=-\frac{1}{2}\left(\theta^{+} \otimes \theta^{-}+\theta^{-} \otimes \theta^{+}\right) \\
& \eta_{+-}=-\frac{1}{2} \\
& \eta^{+-}=-2 \\
& \theta^{0} \wedge \theta^{1}=-\frac{1}{2} \theta^{+} \wedge \theta^{-}, \quad \epsilon_{01}=+1, \quad \epsilon^{01}=-1, \\
& \epsilon_{+-}=-\frac{1}{2}, \quad \epsilon^{+-}=+2, \quad \epsilon_{-}^{-}=+1, \quad \epsilon^{+}=-1, \\
& v^{+}=-2 v_{-}, \quad v^{-}=-2 v_{+}, \quad v_{-}=-\frac{1}{2} v^{+}, \quad v_{+}=-\frac{1}{2} v^{-}, \\
& \|v\|^{2}=-v^{+} v^{-}=-4 v_{+} v_{-}, \quad v^{+} v_{+}=v^{-} v_{-}=\frac{1}{2}\|v\|^{2}, \\
& \square f=\eta^{i j} f_{; i j}=-4 f_{;+-},
\end{aligned}
$$

\section{Appendix C. Cartan's Approach to Geodesics}

Cartan studies geodesics on a manifold $N$ by using the structural equations to study horizontal curves in the bundle of frames [11]. In fact, Cartan often studies families of geodesics via the exponential map generalized to the bundle of frames.

Let $\pi: \mathcal{F}(N) \rightarrow N$ be the orthonormal frame bundle with canonical coframing $\left(\theta^{\mu}, \omega_{\mu \nu}\right)$. It is well known that if $q \in \mathcal{F}(N)$ with $p=\pi(q) \in N$ then a curve in $N$ based at $p$ uniquely lifts to a horizontal curve in $\mathcal{F}(N)$ beginning at $q$. Using his structural equations, Cartan sets up a system of ordinary differential equations satisfied by the horizontal lift of the geodesic. Cartan considers a map $E: \mathbb{R} \times \mathbb{E}^{n} \rightarrow \mathcal{F}(N)$. Fix $q \in N$ and $u \in \mathbb{E}^{n}$ then we have that $E(0, u)=q$ and as $\lambda$ varies we have that $E(\lambda, u)$ will be the horizontal curve with "constant velocity" $u$ :

$$
\begin{aligned}
\theta^{\mu}\left(E_{*}\left(\frac{\partial}{\partial \lambda}\right)\right) & =u^{\mu}, \quad(\text { constant velocity) } \\
\omega_{\mu \nu}\left(E_{*}\left(\frac{\partial}{\partial \lambda}\right)\right) & =0 . \quad \text { (horizontal) }
\end{aligned}
$$


The dual versions of these statements are

$$
\begin{aligned}
E^{*} \theta^{\mu} & =u^{\mu} d \lambda+\vartheta^{\mu}, \\
E^{*} \omega_{\mu \nu} & =\varpi_{\mu \nu},
\end{aligned}
$$

where $\vartheta^{\mu}$ and $\varpi_{\mu \nu}$ are unknown 1-forms on $\mathbb{R} \times \mathbb{E}^{n}$ that are independent of $d \lambda$. Note that on $\mathbb{R} \times \mathbb{E}^{n}$ there are natural global cartesian coordinates $(\lambda, u)$ and differential forms can be assigned a bi-degree $(k, l)$ where $k=0,1$ and $l=$ $0,1, \ldots, n$. For example, $d \lambda$ has bi-degree $(1,0)$ and $\vartheta^{\mu}$ has bi-degree $(0,1)$.

Comment 1. If $N=\mathbb{E}^{n}$ with cartesian coordinates $x$ then the map $\pi \circ E$ is given by $(\lambda, u) \mapsto x^{\mu}=\lambda u^{\mu}$. Note that $d x^{\mu}=u^{\mu} d \lambda+\lambda d u^{\nu}$ and in comparing with (C.2) we see that $\vartheta^{\mu}=\lambda d u^{\mu}$.

Comment 2. On the vector space $\mathbb{R}^{k}$ with standard coordinates $\left(x^{1}, \ldots, x^{k}\right)$, the exterior derivative $d$ acting on a $p$-form $\alpha=\alpha_{J} d x^{J},|J|=p$ (multiindex notation) is simply given by

$$
d \alpha=d x^{i} \wedge \frac{\partial \alpha}{\partial x^{i}},
$$

where

$$
\frac{\partial \alpha}{\partial x^{i}}=\frac{\partial \alpha_{J}}{\partial x^{i}} d x^{J}
$$

Taking the exterior derivatives of (C.2) and using the comments we have

$$
\begin{aligned}
-\varpi_{\mu \nu} \wedge\left(u^{\nu} d \lambda+\vartheta^{\nu}\right) & =d u^{\mu} \wedge d \lambda+d \lambda \wedge \frac{\partial \vartheta^{\mu}}{\partial \lambda}+d u^{\nu} \wedge \frac{\partial \vartheta^{\mu}}{\partial u^{\nu}}, \\
E^{*}\left(-\omega_{\mu \kappa} \wedge \omega_{\kappa \nu}+\frac{1}{2} R_{\mu \nu \rho \sigma} \theta^{\rho} \wedge \theta^{\sigma}\right) & =d \lambda \wedge \frac{\partial \varpi_{\mu \nu}}{\partial \lambda}+d u^{\lambda} \wedge \frac{\partial \varpi_{\mu \nu}}{\partial u^{\lambda}}
\end{aligned}
$$

Identifying the terms that have bi-degree $(1,1)$ we find

$$
\begin{aligned}
\frac{\partial \vartheta^{\mu}}{\partial \lambda} & =d u^{\mu}+\varpi_{\mu \nu} u^{\nu}, \\
\frac{\partial \varpi_{\mu \nu}}{\partial \lambda} & =r_{\mu \nu \rho \sigma} u^{\rho} \vartheta^{\sigma},
\end{aligned}
$$

where $r_{\mu \nu \rho \sigma}=E^{*} R_{\mu \nu \rho \sigma}$. The initial conditions on these differential equations are $\left.\vartheta^{\mu}\right|_{\lambda=0}=0$ and $\left.\varpi_{\mu \nu}\right|_{\lambda=0}=0$. This follows from the condition that $E(0, u)=q$ for all $u$, see for example Comment 1 . 
Equations (C.4) can be combined into a second order differential equation

$$
\frac{\partial^{2} \vartheta^{\mu}}{\partial \lambda^{2}}=r_{\mu \nu \rho \sigma} u^{\nu} u^{\rho} \vartheta^{\sigma}
$$

with initial conditions $\left.\vartheta^{\mu}\right|_{\lambda=0}=0$ and $\left.\left(\partial \theta^{\mu} / \partial \lambda\right)\right|_{\lambda=0}=d u^{\mu}$. This equation is Cartan's equation for a Jacobi vector field. It tells you how the coframe changes along the horizontal lift of a geodesic.

C.1. Holonomy and Symmetric Spaces. Using the methods of the previous section it is easy to understand the basic properties of symmetric spaces. Choose a point $q \in \mathcal{F}(M)$, let $\Gamma_{q}$ be the holonomy group at $q$ and let $\Phi_{q} \subset \mathcal{F}(M)$ be the set of all points in the frame bundle that are connected to $q$ by a piecewise differentiable horizontal curve. The basic theorem is that the holonomy bundle $\Phi_{q}$ is a sub-bundle of $\mathcal{F}(M)$ with structure group $\Gamma_{q} \subset \mathrm{SO}(n)$ and that $M=\Phi_{q} / \Gamma_{q}$, see $[7]$.

Next we show that the riemannian connection restricted to the holonomy bundle is a $\Gamma_{q}$-connection. To do this we write the Lie algebra

$$
\mathfrak{s o}(n)=\mathfrak{g} \oplus \mathfrak{h}
$$

where $\mathfrak{g}$ is the Lie algebra of $\Gamma_{q}$ and $\mathfrak{h}$ is a complementary subspace. Under this decomposition the Cartan structural equations become

$$
\begin{aligned}
d \omega^{\alpha} & =-\frac{1}{2} f^{\alpha}{ }_{\beta \gamma} \omega^{\beta} \wedge \omega^{\gamma}-f_{\beta c}^{\alpha} \omega^{\beta} \wedge \omega^{c}-\frac{1}{2} f_{b c}^{\alpha} \omega^{b} \wedge \omega^{c}+\frac{1}{2} R^{\alpha}{ }_{\mu \nu} \theta^{\mu} \wedge \theta^{\nu}, \\
d \omega^{a} & =-\frac{1}{2} f^{a}{ }_{\beta \gamma} \omega^{\beta} \wedge \omega^{\gamma}-f^{a}{ }_{\beta c} \omega^{\beta} \wedge \omega^{c}-\frac{1}{2} f_{b c}^{a} \omega^{b} \wedge \omega^{c}+\frac{1}{2} R_{\mu \nu}^{a} \theta^{\mu} \wedge \theta^{\nu}, \\
d \theta^{\mu} & =-A^{\mu}{ }_{\nu \alpha} \omega^{\alpha} \wedge \theta^{\nu}-A^{\mu}{ }_{\nu a} \omega^{a} \wedge \theta^{\nu} .
\end{aligned}
$$

In the above the indices $a, b, c$ refer to $\mathfrak{g}$ and $\alpha, \beta, \gamma$ refer to $\mathfrak{h}$. The $f$ are the structure constants for $\mathfrak{s o}(n)$ adapted to the decomposition (C.6) and the $A$ are other constants associated to the same decomposition ${ }^{13}$. The holonomy bundle is a sub-bundle that solves the equation $\omega^{\alpha}=0$. The reason is that a basis for $T_{q} \Phi_{q}$ is $\left\{e_{\mu}\right\} \cup\left\{e_{a}\right\}$, i.e., need the horizontal curves that are used to construct the holonomy bundle and also need the holonomy Lie algebra. The Frobenius theorem requires $f_{b c}^{\alpha}=0$ and $R^{\alpha}{ }_{\mu \nu}=0$ for an integrable distribution. This means that $\mathfrak{g}$ is a subalgebra of $\mathfrak{s o}(n)$ as required and there is no curvature in

\footnotetext{
${ }^{13}$ These are really associated with the decomposition of the basic representation of $\mathfrak{s o}(n)$ in terms of the decomposition (C.6).
} 
the "h-direction". Restricting to the holonomy sub-bundle $\Phi_{q}$ we have structural equations

$$
\begin{aligned}
d \omega^{a} & =-\frac{1}{2} f_{b c}^{a} \omega^{b} \wedge \omega^{c}+\frac{1}{2} R_{\mu \nu}^{a} \theta^{\mu} \wedge \theta^{\nu}, \\
d \theta^{\mu} & =-A_{\nu a}^{\mu} \omega^{a} \wedge \theta^{\nu} .
\end{aligned}
$$

These equation tell us that the restriction of the connection to the holonomy bundle is a $\Gamma_{q}$-connection $^{14}$.

How do symmetric spaces arise from this viewpoint ${ }^{15}$ ? If the curvature is covariantly constant then it is a constant function on the holonomy sub-bundle. The reason is that $d R=-\omega \cdot R+\left(\nabla_{\lambda} R\right) \theta^{\lambda}=-\omega \cdot R$ which vanishes along a horizontal curve. Consequently $R_{\mu \nu \rho \sigma}$ must be a constant function on the holonomy sub-bundle $\Phi_{q}$. This means that $R^{a}{ }_{\mu \nu}$ are constant and therefore equations (C.8) are the Maurer-Cartan equations for a Lie group $G$. Therefore $\Phi_{q} \approx G$ and $M=G / \Gamma_{q}$. There is a stronger statement we can make. The Maurer-Cartan equations (C.8) admit a symmetry $\omega \rightarrow \omega$ and $\theta \rightarrow-\theta$. This is the famous Cartan involution that leads to symmetric Lie algebras and associated symmetric spaces. The reason for "symmetric" may be see in (C.5). Note that $r_{\mu \nu \rho \sigma} u^{\nu} u^{\sigma}$ are constant and therefore $\lambda \rightarrow-\lambda$ is a symmetry of the differential equation. This means that by integrating (C.5) to construct the metric we have an isometry between the point at time $\lambda$ and the one at $-\lambda$. This is the Cartan local isometry in a symmetric space ${ }^{16}$.

\section{REFERENCES}

[1] H. Stephani, D. Kramer, M. MacCallum, C. Hoenselaers, and E. Herlt, Exact solutions of Einstein's field equations. Cambridge Monographs on Mathematical Physics. Cambridge University Press, Cambridge, second ed., 2003.

[2] S. Carroll, Spacetime and geometry: an introduction to general relativity. Addison Wesley, 2004.

[3] R. d'Inverno, Introducing Einstein's relativity. The Clarendon Press, Oxford University Press, New York, 1992.

\footnotetext{
${ }^{14}$ These arguments generalize to the holonomy bundle of a generic bundle not just the frame bundle.

${ }^{15}$ I ignore some issues of connected component, etc.

${ }^{16}$ Note that we can use (C.5) to conclude the converse. If we have the local isometry about any point then (C.5) must be even under $\lambda \rightarrow-\lambda$ and therefore $r_{\mu \nu \rho \sigma}$ must be an even function and therefore the derivative vanishes at $\lambda=0$. This being true at all points implies that $\nabla R=0$.
} 
[4] C. W. Misner, K. S. Thorne, and J. A. Wheeler, Gravitation. W. H. Freeman and Co., San Francisco, Calif., 1973.

[5] S. W. Hawking and G. F. R. Ellis, The large scale structure of space-time. Cambridge University Press, London, 1973. Cambridge Monographs on Mathematical Physics, No. 1.

[6] R. M. Wald, General relativity. University of Chicago Press, Chicago, IL, 1984.

[7] S. Kobayashi and K. Nomizu, Foundations of differential geometry. Vol I. Interscience Publishers, a division of John Wiley \& Sons, New York-London, 1963.

[8] B. O'Neill, "The fundamental equations of a submersion," Michigan Math. J. 13 (1966) 459-469.

[9] B. O’Neill, Semi-Riemannian Geometry. Academic Press, 1983.

[10] F. W. Warner, Foundations of Differentiable Manifolds and Lie Groups. Scott, Foresman and Co., 1971.

[11] É. Cartan, Leçons sur la Géométrie des Espaces de Riemann. Gauthier-Villars, Paris, 1946. 2 d ed.

[12] B. Carter, "Black hole equilibrium states," in Black Holes, C. DeWitt and B. DeWitt, eds., pp. 57-214. Gordon and Breach Science Publishers, 1973. Lectures presented at the Les Houches Summer School in August 1972.

[13] J. Milnor, Morse theory. Based on lecture notes by M. Spivak and R. Wells. Annals of Mathematics Studies, No. 51. Princeton University Press, Princeton, N.J., 1963.

Orlando Alvarez

Department of Physics

University of Miami

P.O. Box 248046

Coral Gables, FL 33124 USA

E-mail: oalvarez@miami.edu 